\title{
Prevention of hepatocarcinogenesis and increased susceptibility to acetaminophen- induced liver failure in transaldolase-deficient mice by $\mathrm{N}$-acetylcysteine
}

\begin{abstract}
Robert Hanczko,1 David R. Fernandez,1,2 Edward Doherty, ${ }^{1}$ Yueming Qian, ${ }^{1}$ Gyorgy Vas, ${ }^{1}$ Brian Niland, ${ }^{1}$ Tiffany Telarico, ${ }^{1,2}$ Adinoyi Garba, ${ }^{1}$ Sanjay Banerjee, ${ }^{1}$ Frank A. Middleton, ${ }^{3}$ Donna Barrett, ${ }^{4}$ Maureen Barcza, ${ }^{4}$ Katalin Banki, ${ }^{4}$ Steve K. Landas, ${ }^{4}$ and Andras Perl1,2
\end{abstract}

1Department of Medicine, ${ }^{2}$ Department of Microbiology and Immunology, ${ }^{3}$ Department of Neuroscience and Physiology, and ${ }^{4}$ Department of Pathology, College of Medicine, State University of New York, Syracuse, New York, USA.

\begin{abstract}
Although oxidative stress has been implicated in acute acetaminophen-induced liver failure and in chronic liver cirrhosis and hepatocellular carcinoma (HCC), no common underlying metabolic pathway has been identified. Recent case reports suggest a link between the pentose phosphate pathway (PPP) enzyme transaldolase (TAL; encoded by TALDO1) and liver failure in children. Here, we show that Taldo1 ${ }^{-/-}$and $\mathrm{Taldo}^{+/-}$mice spontaneously developed HCC, and Taldo1 ${ }^{-/-}$mice had increased susceptibility to acetaminophen-induced liver failure. Oxidative stress in Taldo1 $1^{-/}$livers was characterized by the accumulation of sedoheptulose 7-phosphate, failure to recycle ribose 5-phosphate for the oxidative PPP, depleted NADPH and glutathione levels, and increased production of lipid hydroperoxides. Furthermore, we found evidence of hepatic mitochondrial dysfunction, as indicated by loss of transmembrane potential, diminished mitochondrial mass, and reduced ATP/ADP ratio. Reduced $\beta$-catenin phosphorylation and enhanced $c$-Jun expression in Taldo1 ${ }^{-/-}$livers reflected adaptation to oxidative stress. Taldo1 ${ }^{-/}$hepatocytes were resistant to CD95/Fas-mediated apoptosis in vitro and in vivo. Remarkably, lifelong administration of the potent antioxidant $\mathrm{N}$-acetylcysteine (NAC) prevented acetaminophen-induced liver failure, restored Fas-dependent hepatocyte apoptosis, and blocked hepatocarcinogenesis in Taldo1 ${ }^{-/-}$mice. These data reveal a protective role for the TAL-mediated branch of the PPP against hepatocarcinogenesis and identify NAC as a promising treatment for liver disease in TAL deficiency.
\end{abstract}

\section{Introduction}

Hepatocellular carcinoma (HCC) is the fifth most common cancer and the third leading cause of cancer-related mortality worldwide (1). More than $80 \%$ of European and US cases of HCC develop in cirrhotic livers (2). The prevalence of chronic liver disease and cirrhosis is approximately 400,000 , or $0.15 \%$, in the US. Despite recognition of alcohol and hepatitis $\mathrm{C}$ and $\mathrm{B}$ viruses as etiologic agents, the cause of liver cirrhosis in most HCC patients is unknown (3). Elevated serum levels of $\alpha$-fetoprotein (AFP) correlate with development of HCC, and it is used as a screening test in cirrhotic patients (4). Hepatocyte transformation is thought to occur in the setting of chronic inflammation, regeneration, and oxidative stress, leading to DNA damage and activation of oncogenes and inactivation of tumor suppressor genes (5). There is growing evidence for the involvement of the CD95/Fas cell death

Conflict of interest: The authors have declared that no conflict of interest exists. Nonstandard abbreviations used: AFP, $\alpha$-fetoprotein; APAP, acetaminophen; AR, aldose reductase; CAT, catalase; G6PD, glucose 6-phosphate dehydrogenase; GSH, glutathione; HCC, hepatocellular carcinoma; 4-HNE, 4-hydroxynonenal; LC-MS/MS, liquid chromatography coupled to tandem mass spectroscopy; LPO, lipid hydroperoxide; MDA, malondialdehyde; NAC, N-acetylcysteine; NADP, NAD phosphate; NAFLD, nonalcoholic fatty liver disease; NASH, nonalcoholic steatohepatitis; PCNA, proliferating cell nuclear antigen; PPP, pentose phosphate pathway; ROI, reactive oxygen intermediate; R5P, ribose 5-phosphate; S7P, sedoheptulose 7-phosphate; TAL, transaldolase; X5P, xylulose 5-phosphate; $\Delta \psi_{\mathrm{m}}$, mitochondrial transmembrane potential.

Citation for this article: J. Clin. Invest. 119:1546-1557 (2009). doi:10.1172/JCI35722. receptor-initiated apoptosis pathway in physiological regulation of hepatocyte turnover and hepatocarcinogenesis (6-10). Apoptosis resistance and uncontrolled proliferation of hepatocytes is associated with increased expression of c-Jun, a redox-sensitive transcription factor $(11,12)$. Oxidative stress is also involved in toxicity by acetaminophen (APAP; acetyl-p-aminophenol, also known as Tylenol; McNeil-PPC Inc.), which is the leading cause of acute liver failure (13). APAP is metabolized by hepatic cytochrome $\mathrm{P} 450$ into a strongly oxidizing intermediate ( $\mathrm{N}$-acetyl-para benzoquinone imine, NAPQI), which is detoxified at the expense of reduced glutathione (GSH) (14). To date, common genetic factors have yet to be identified that predispose either to cirrhosis and hepatocarcinogenesis or to APAP-induced acute liver failure.

Homozygous mutations of transaldolase (TAL; encoded by TALDO1) have been reported in a 9-year-old girl with liver cirrhosis (15), a newborn girl who died of liver failure at the age of 18 days (16), and 4 siblings with multiorgan disease, hydrops fetalis, and genitourinary malformations (17). Because all 6 patients were offspring of consanguineous parents, mutations in genes other than TALDO1 could have influenced the wide spectrum of disease manifestations. To investigate the role of TAL in mammalian development, and specifically in liver disease, we inactivated the TAL locus in mice. Mice deficient in Taldo1 develop normally, with the exception of sperm dysmotility (18). Taldo $1^{+/-}$and Taldo $1^{-/-}$mice are 27and 79-fold more likely to spontaneously develop liver cirrhosis and nodular dysplasia, respectively, than their wild-type Taldo1 $1^{+/+}$ 
A

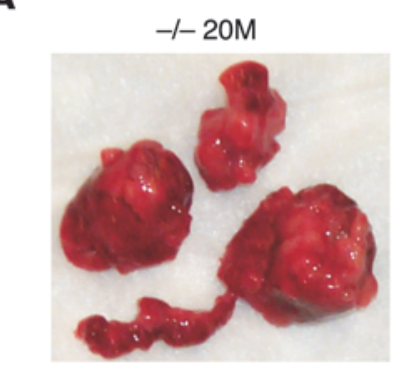

B

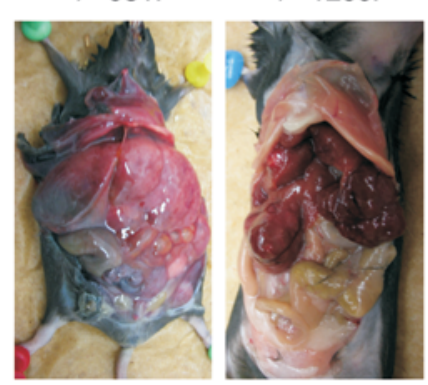

Nodular dysplasia and inflammation
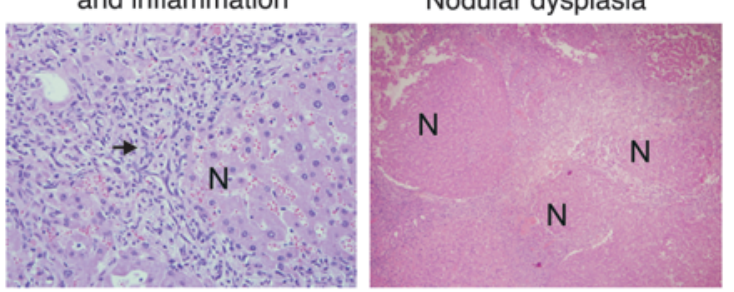

Nodular dysplasia (Klatskin)

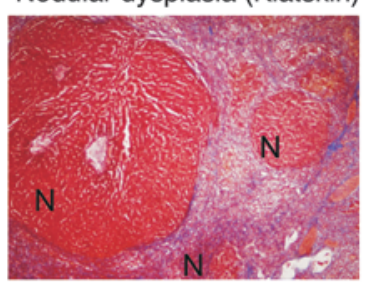

Hepatoma with mitotic figures
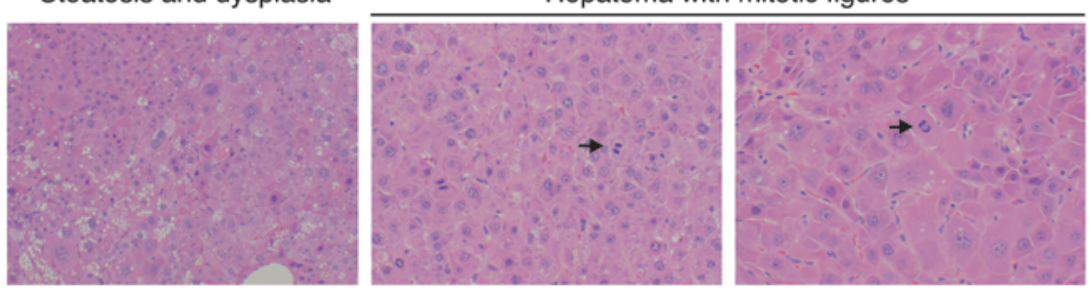

\section{Figure 1}

Spontaneous development of dysplastic nodules, cirrhosis, and HCC in Taldo1-/- mice. (A) Dysplastic nodules and cirrhosis in Taldo1-/- (-/-) mice. Macroscopic and microscopic images of 52-week-old Taldo $1^{-1-}$ mouse $20 \mathrm{M}$ stained with H\&E and Klatskin trichrome stain. Arrow denotes inflammatory cells surrounding nodules $(\mathrm{N})$. Klatskin trichrome staining of the liver showed interstitial deposition of collagen (blue). Hepatocytes within the nodules were avidly stained red. Original magnification, left to right: $\times 100, \times 40, \times 40$. (B) Macroscopic images of HCC in a 74-week-old (931F) and an 82 week-old (1266F) Taldo1-/- mice, and microscopic images of steatosis, dysplasia, mitotic figures (arrows), and hepatoma in 74-week-old Taldo1-/- mouse 931F. Original magnification, left to right: $\times 100, \times 100, \times 200$.

littermates. We found that HCC was the most common malignancy and leading cause of death in both Taldo $1^{+/-}$and Taldo $1^{-/-}$mice. Paradoxically, Taldo1 $1^{-1-}$ mice were resistant to CD95-dependent hepatocyte apoptosis and were highly susceptible to APAP-induced liver cell necrosis. Oral administration of $\mathrm{N}$-acetylcysteine (NAC) blocked APAP susceptibility, restored Fas-dependent apoptosis, and prevented (a) dephosphorylation of $\beta$-catenin, (b) activation of c-Jun, and (c) development of cirrhosis and hepatocarcinogenesis in Taldo1 $1^{-/-}$mice.

\section{Results}

Spontaneous development of steatosis, steatohepatitis, cirrhosis, dysplasia, and HCC in the livers of Taldo1 $1^{-/-}$and $\mathrm{Taldo}^{+/-}$mice. To characterize the Taldo1 $1^{-/-}$phenotype, we analyzed 102 litters of crosses between Taldo $1^{+/-}$heterozygotes, each litter consisting of at least 1 Taldo ${ }^{+/+}$, Taldo $1^{+/-}$, and Taldo1 $1^{-/-}$animal. When 1 litter member became ill, the entire litter was autopsied. HCC was the leading cause of death in both Taldo1+- (39 of 229; 17\%) and Taldo1/-- mice (45 of 97; 46\%), in contrast to Taldo $1^{+/+}$littermates ( 1 of $102 ; 1 \%$; Figure 1 and Table 1 ). The normal liver architecture (i.e., typical hexagonal central-portal relationships) was distorted in Taldo $1^{-/-}$livers, which showed collagen deposition resembling cirrhosis as well as the formation of dysplastic nodules, cellular atypia, anisonucleosis, mitotic figures, increased nuclear to cytoplasmic ratio, and large cell change (Figure 1 and ref. 19). Rates of anisonucleosis and nodular or large cell dysplasia, based on established criteria (19), were markedly increased in both Taldo1 $1^{+-}$and Taldo $1^{-/-}$mice $(P<0.0001$; Table 1$)$.

To assess pathological changes that preceded liver cirrhosis and dysplasia, 10- to 12-week-old litters were examined. We observed the formation of microvesicular and macrovesicular lipid droplets indicative of steatosis or nonalcoholic fatty liver disease (NAFLD), hepatocyte ballooning with Mallory hyalin, expansion of lipid-containing perisinusoidal Ito cells, and inflammatory changes resembling nonalcoholic steatohepatitis (NASH; Figure 2 and ref. 20). Similar to patients with fatty liver (20), Taldo $1^{-/-}$mice had diminished glucose tolerance (Supplemental Figure 1). Electron microscopy showed accumulation of lipid droplets and also diminished size and numbers of mitochondria in Taldo1 $1^{-/-}$ hepatocytes (Supplemental Figure 2). Hepatocyte death was evaluated by serum levels of liver enzymes and TUNEL staining of hepatocytes in 4-month-old and 8-week-old littermates. Relative to Taldo $1^{+/+}$control littermates $(145 \pm 21 \mathrm{IU} / \mathrm{l})$, serum aspartate aminotransferase (AST) levels were increased in Taldo1 ${ }^{-/-}$mice at the age of 4 months $(224 \pm 37 \mathrm{IU} / 1 ; P=0.034)$. Serum alanine ami-

\section{Table 1}

Frequency of malignant tumors, HCC, and liver cell dysplasia

\begin{tabular}{lcccc} 
Frequency & $\begin{array}{c}\text { Taldo1+/+ } \\
(\boldsymbol{n = 1 0 2 )}\end{array}$ & $\begin{array}{c}\text { Taldo1+/- } \\
(\boldsymbol{n}=\mathbf{2 2 9})\end{array}$ & $\begin{array}{c}\text { Taldo1-/- } \\
(\boldsymbol{n}=\mathbf{9 7 )}\end{array}$ & $\boldsymbol{P A}^{\mathrm{A}}$ \\
All malignant tumors & $6 / 96$ & $74 / 155$ & $55 / 42$ & $<0.0001$ \\
HCC only & $1 / 101$ & $39 / 190$ & $45 / 52$ & $<0.0001$ \\
Liver dysplasiaC $^{\mathrm{C}}$ & $1 / 101$ & $62 / 167$ & $77 / 20$ & $<0.0001$ \\
\hline
\end{tabular}

Values denote ratio of presence/absence. ${ }^{\mathrm{A}} \mathrm{Ta}$ aldo $1^{-/-}$versus littermate control mice. $P$ values were determined by $\chi^{2}$ test. BHCC, lymphomas, carcinomas, sarcomas. 'Large cell change dysplasia, nodular dysplasia; includes HCC. 
A

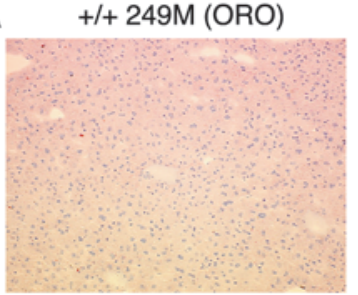

B

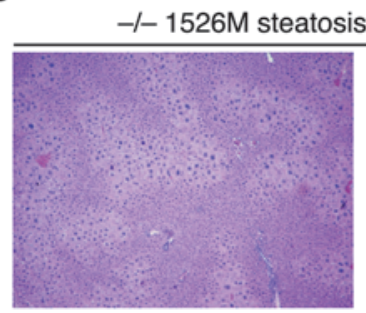

C

$$
-/-735 \mathrm{M} \text { inflammation }
$$

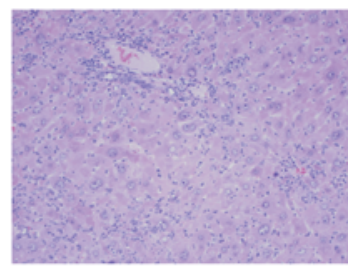

$-/-246 \mathrm{M}$ (ORO)

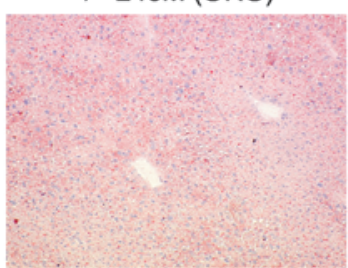

anisonucleosis

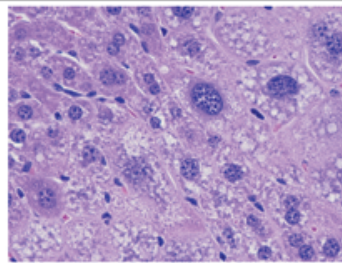

$-/-735 \mathrm{M}$ large cell change dysplasia

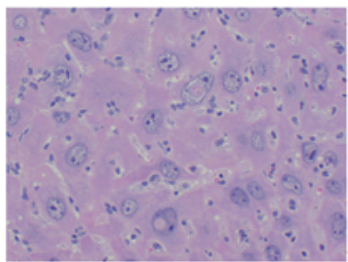

$+/+239 \mathrm{~F}(\mathrm{ORO})$

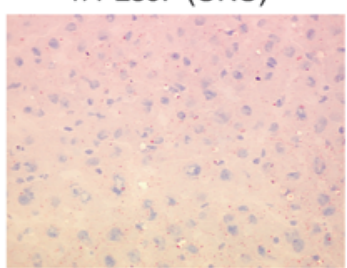

+/- 979F steatosis

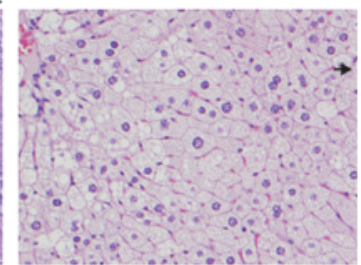

- - 288M dysplasia with Ito cells

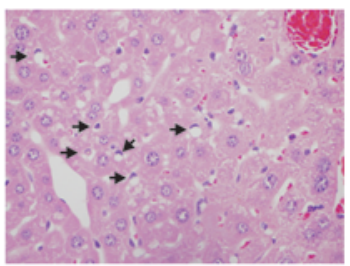

$-1-244 \mathrm{~F}$ (ORO)

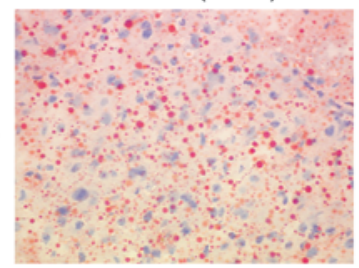

$+/-618 \mathrm{M}$ steatosis and Mallory bodies

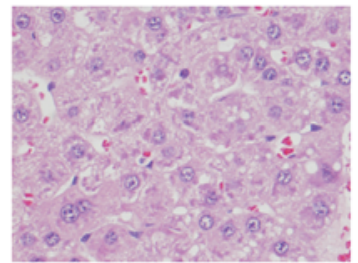

-/- 44F inflammation with Ito cells (GFAP)

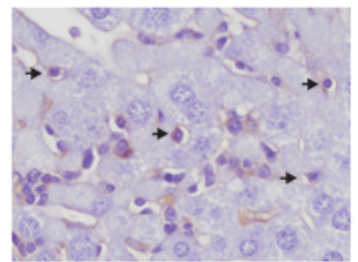

Figure 2

NAFLD and NASH with dysplasia in 10- to 12-week-old Taldo1-/- mice. (A) Detection of lipid droplets with oil-red-O (ORO) staining in frozen liver sections of Taldo $1^{-/-}$and Taldo $1^{+/+}(+/+)$littermates. Original magnification, left to right: $\times 100, \times 100, \times 400, \times 400$. (B) Centrolobular, zonal, or diffuse steatosis and Mallory bodies in Taldo $1^{-/}$and Taldo $1^{+-}(+/-)$mice. Original magnification, left to right: $\times 40, \times 400, \times 400, \times 400$. (C) Steatosis, inflammation, liver cell dysplasia with large cell change, and expansion of fat-storing hepatic stellate or Ito cells (arrows). Ito cells were also identified by expression of glial fibrillary acidic protein (GFAP). Original magnification, left to right: $\times 100, \times 400, \times 400, \times 600$.

notransferase (ALT) levels were similar in Taldo1 ${ }^{+++}(64 \pm 13 \mathrm{IU} / \mathrm{l})$ and Taldo $1^{-/-}$mice $(78 \pm 24 \mathrm{IU} / 1 ; P=0.632)$. This elevated AST relative to ALT was consistent with the development of cirrhosis in the context of chronic hepatitis (21) and NAFLD (22). The number of TUNEL-positive apoptotic hepatocytes increased in 8-weekold Taldo1 $1^{-/}(6.0 \% \pm 0.7 \% ; P=0.014)$ and Taldo $1^{+/-}(3.6 \% \pm 0.3 \%$; $P=0.012)$ mice relative to Taldo $1^{+/+}$controls $(0.2 \% \pm 0.1 \%)$. Thus, liver cell injury of Taldo1 $1^{-/}$mice was detectable earlier by TUNEL staining than by elevation of serum AST. Cell proliferation was evaluated by proliferating cell nuclear antigen (PCNA) staining of livers (Supplemental Figure 3). The number of PCNA-positive nuclei per 1,000 hepatocytes increased in 8-week-old Taldo1 $1^{-/}(20.9 \pm 5.8$ nuclei; $P=0.0093)$ and Taldo $1^{+/-}(2.4 \pm 0.2$ nuclei; $P=0.0058)$ mice relative to Taldo $1^{+/+}$controls ( $1.17 \pm 0.3$ nuclei). PCNA staining was markedly elevated in hepatomas (587.0 \pm 140.8 nuclei) compared with Taldo1 $1^{-/-}$livers $(20.9 \pm 5.8$ nuclei; $P=0.0011)$.

Metabolic consequences of TAL deficiency are characterized by depleted $N A D P H$ and GSH, diminished NO production, lost mitochondrial transmembrane potential and mitochondrial mass, and reduced ATP/ADP ratio. The metabolism of glucose through the pentose phosphate pathway (PPP) provides ribose 5-phosphate (R5P) for the synthesis of nucleotides and NADPH, a reducing equivalent for biosynthetic reactions and for maintenance of GSH in its reduced state $(23,24)$. To assess the consequences of TAL deficiency on the PPP, we determined the levels of PPP sugars and nucleotides by liquid chromatography coupled to tandem mass spectroscopy (LC-MS/ MS) (25). The unique TAL substrate sedoheptulose 7-phosphate (S7P) accumulated 17-fold in Taldo1 $1^{-/}$livers (Figure 3). The levels of C5 sugar phosphates R5P and xylulose 5-phosphate (X5P) and C5-polyols D-arabitol and D-ribitol also increased (Figure $3 \mathrm{~A})$. ADP and ATP levels increased, the latter to a lesser extent, which caused a reduced ATP/ADP ratio (Figure $3 \mathrm{~A}$ ). NADPH and the NADPH/NAD phosphate (NADPH/NADP) ratio fell in both Taldo $1^{+/-}$and Taldo $1^{-/-}$livers, whereas NADH was only reduced in Taldo1 $1^{-/}$livers (Figure 3B). By HPLC (18), GSH levels in the livers of Taldo1 $1^{-1-}$ mice were reduced $(3.502 \pm 0.174 \mathrm{nmol} / \mathrm{mg}$ tissue; $n=9)$ in comparison to Taldo $1^{+/+}$littermates $(4.254 \pm 0.193 \mathrm{nmol} /$ mg tissue; $n=9 ; P<0.0001)$. Flow cytometry also showed diminished GSH content in Taldo1 ${ }^{-/-}(-29.7 \% ; P=0.0008)$ and Taldo $1^{+/-}$ hepatocytes $(-21.3 \% ; P=0.005)$ with respect to Taldo $1^{+/+}$controls (Figure 4). The low NADPH and GSH levels thus provided the metabolic basis for oxidative stress.

The oxidation-reduction equilibrium of pyridine nucleotides (i.e., the combined NADH/NAD and NADPH/NADP ratios) and GSH regulate the mitochondrial transmembrane potential $\left(\Delta \psi_{\mathrm{m}}\right)(26)$. Mitochondria play crucial roles in cell death signal processing by hepatocytes (27). Both $\Delta \psi_{\mathrm{m}}$ and mitochondrial mass were reduced in hepatocytes of 8- to 10-week-old Taldo1-/- mice (Figure 4). 

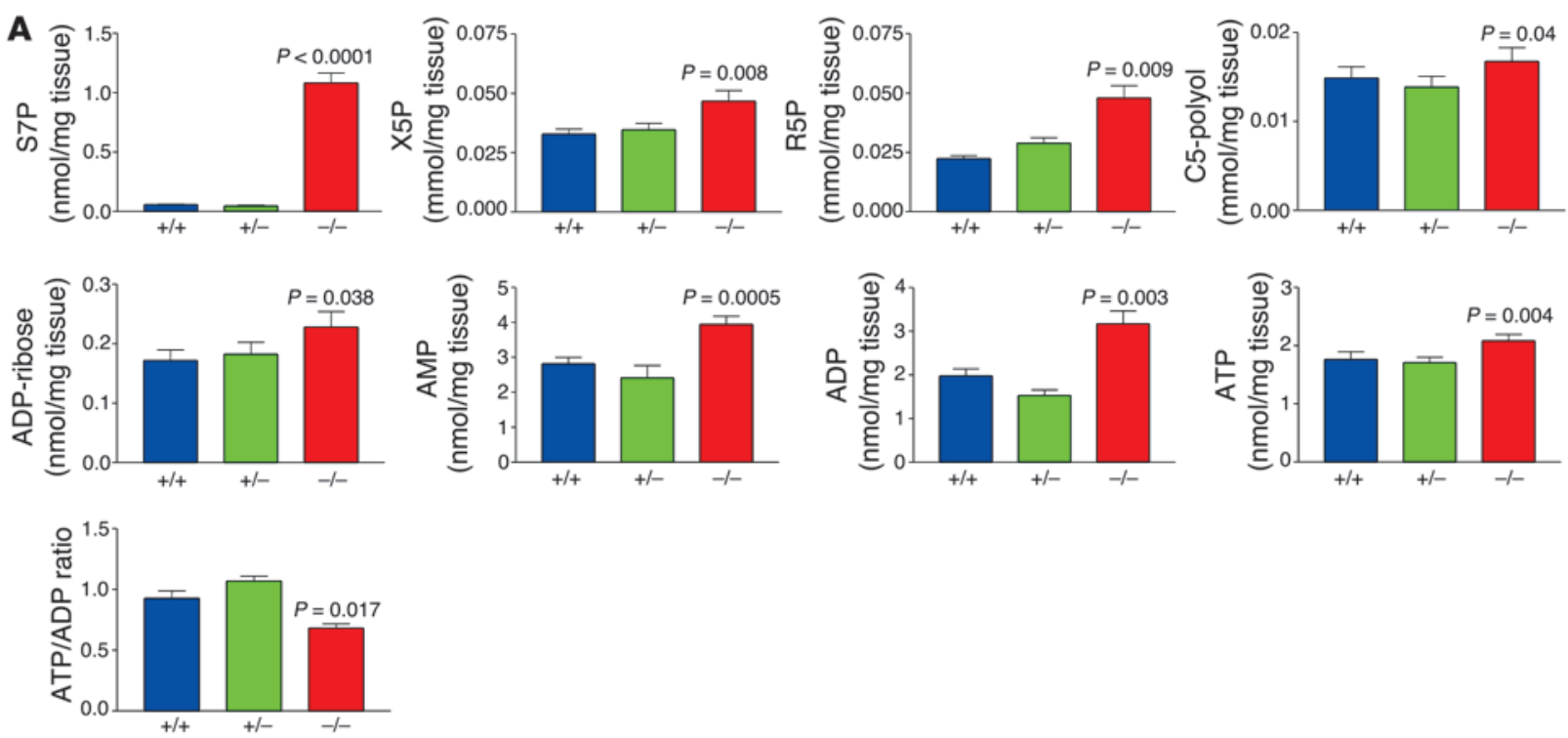

B
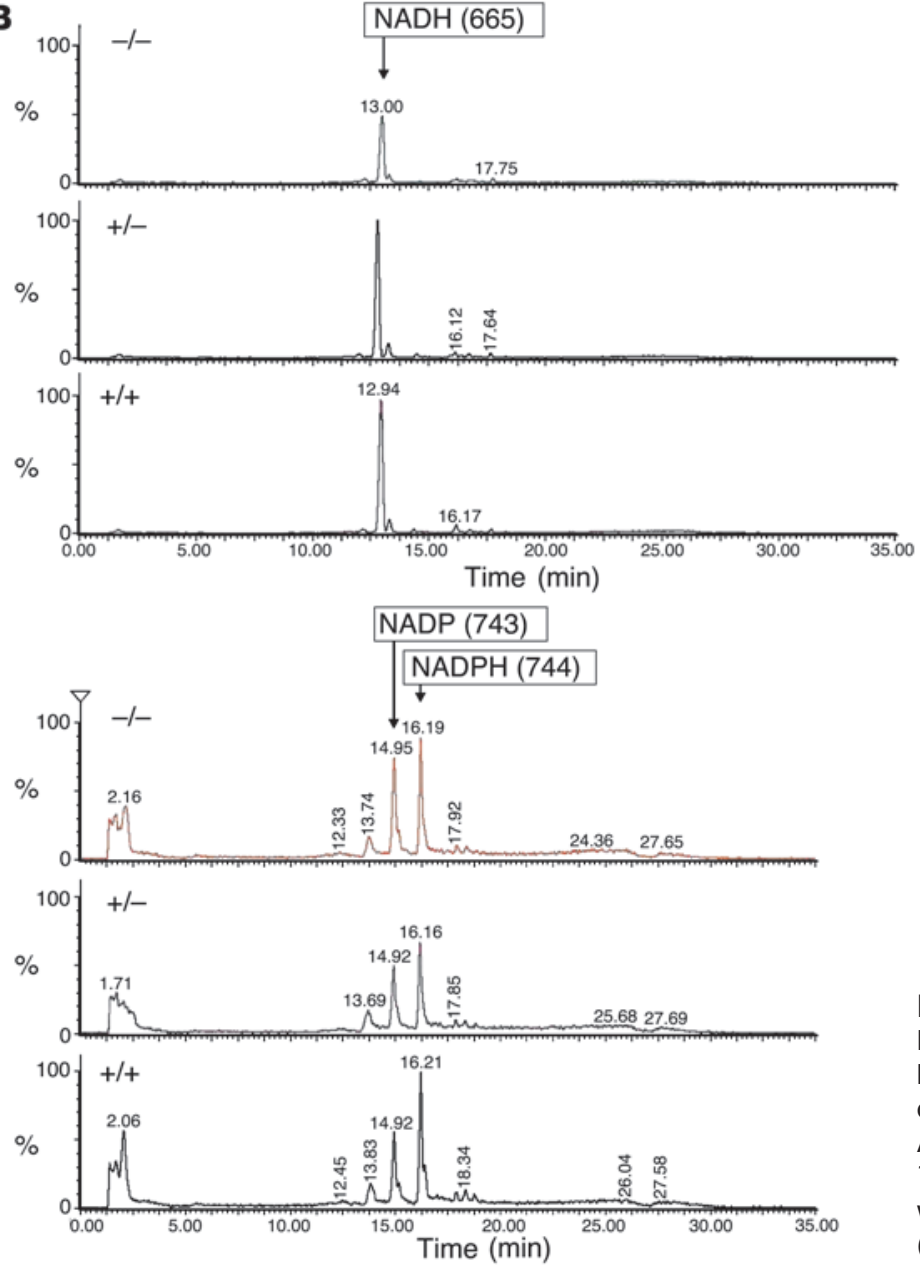
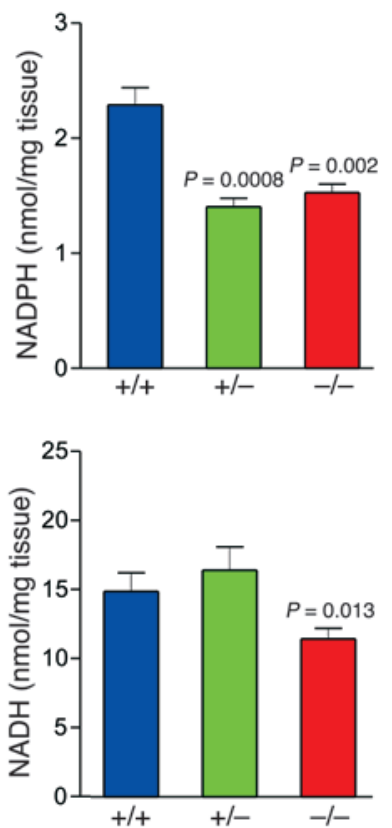

\section{Figure 3}

LC-MS/MS analysis of PPP sugars and nucleotides in the liver. (A) Cumulative analysis of S7P, X5P, R5P, C5-polyol, ADP-ribose, AMP, ADP, and ATP levels as well as the ATP/ADP ratio in the livers of 14 sets ( 6 male, 8 female) of

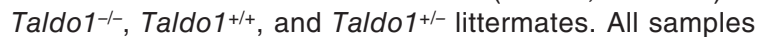
were analyzed after methanol/water extraction by LC-MS/MS (25). Sugar phosphates and nucleotides were detected in the same sample. (B) Quantitative recovery of NADH and NADPH was achieved as described in Methods. This method allowed parallel detection of NADH, NADP, and NADPH, as shown by representative MS/MS chromatograms in liver samples from female littermates. Cumulative analysis of NADPH and NADH levels in 12 female littermates $5-6$ weeks of age is shown at right. $P$ values compared with $T$ aldo $1^{+/+}$mice are shown. 


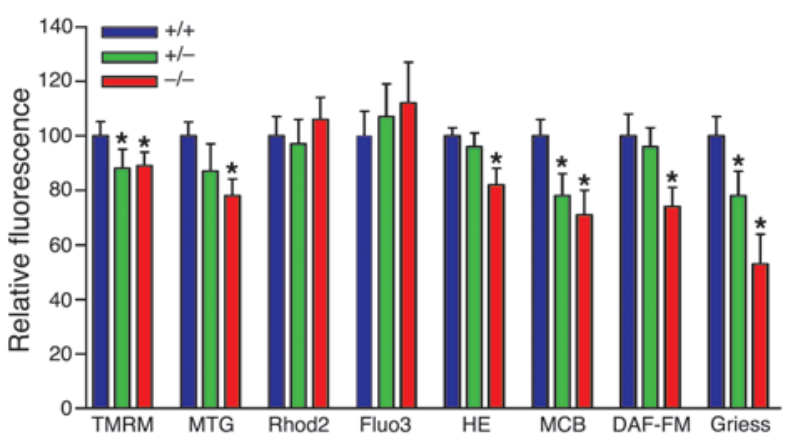

Production of NO, which is required for mitochondrial biogenesis (28), was diminished in Taldo1 $1^{-/-}$hepatocytes. In accordance with the reduced mitochondrial mass and $\Delta \psi_{\mathrm{m}}$, the production of reactive oxygen intermediates (ROIs) was also diminished. Depletion of intracellular GSH levels in excess of the reduction in ROI production and increased SOD, catalase (CAT), and GSH peroxidase (GPx) activities were all consistent with oxidative stress (Supplemental Table 1). Though transketolase was unchanged, glucose 6phosphate dehydrogenase (G6PD) activity was reduced in Taldo1/-livers (Supplemental Table 1). Oxidative stress was also evidenced by increased levels of lipid hydroperoxide (LPO), malondialdehyde (MDA), and 4-hydroxynonenal (4-HNE) adducted proteins in Taldo1 $1^{-/}$livers (Supplemental Figure 4).

Increased expression of c-Jun, AFP, and aldose reductase in Taldo $1^{-/-}$livers and hepatomas. Hepatocarcinogenesis occurs during a multistep process of genetic changes reflecting adaptation to oxidative stress (27, 29). To delineate the changes in gene expression underlying tumor development in TAL deficiency, we performed microarray analysis of microscopically normal livers of 5-week-old litters and litters sacrificed at the time of HCC development by 1 member. Analysis of

\section{Figure 4}

Flow cytometry analysis of mitochondrial function and NO production by isolated hepatocytes. Mouse hepatocytes were isolated from 8- to

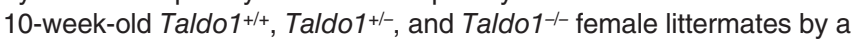
2-step perfusion procedure as described in Methods. $\Delta \psi_{\mathrm{m}}$ by TMRM, mitochondrial mass by MitoTracker Green (MTG), mitochondrial and cytoplasmic $\mathrm{Ca}^{2+}$ levels by Rhod-2 and Fluo-3, ROI production by H\&E, and NO production by DAF-FM fluorescence were assessed in annexin Vnegative cells (72). GSH levels were assessed by monochlorobimane (MCB) fluorescence. NO levels were also assessed by the Griess reaction. Data are mean of 4 or more independent experiments normalized to Taldo1 ${ }^{+/+}$hepatocyte values, set as $100 \%$. ${ }^{*} P<0.05$ versus Taldo $1^{+/+}$.

5-week-old litters revealed greater than 2 -fold enhanced expression

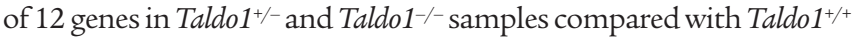
controls (Supplemental Table 2). Remarkably, expression of c-Jun, a redox-sensitive transcription factor and promoter of hepatocyte proliferation (30), increased 5.7- and 8.0-fold in Taldo1 ${ }^{+/-}$and Taldo1 $1^{-/-}$ livers, respectively. Overexpression of c-Jun was confirmed by Western blot analysis (Figure 5). Protein levels of c-Jun in hepatomas from Taldo $1^{-/}$mice aged 15 months or older were similar to levels in microscopically tumor-free livers from 5 -week-old Taldo $1^{-/-}$mice, which suggests that c-Jun activation is an early event of hepatocarcinogenesis (Figure 5). Microarray studies of 6 Taldo1 $1^{-/}$hepatomas revealed overexpression of 30 genes (Supplemental Table 3), including AFP, which is regulated by c-Jun (31) and serves as a biomarker of HCC development in liver disease patients (4), and aldose reductase (AR), which is also redox controlled and has been implicated in hepatocarcinogenesis (32). Western blot analysis confirmed the increased expression of AFP and AR in Taldo $1^{-/-}$hepatomas over agematched tumor-free Taldo $1^{-/-}$livers (Figure 5).

Hypophosphorylation of $\beta$-catenin in Taldo1 $1^{-1}$ - livers and accumulation in the nuclei of Taldo1 $1^{-1-}$ hepatomas. The $\beta$-catenin pathway has been

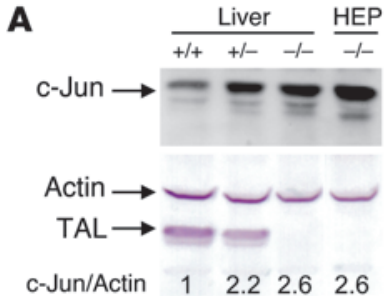

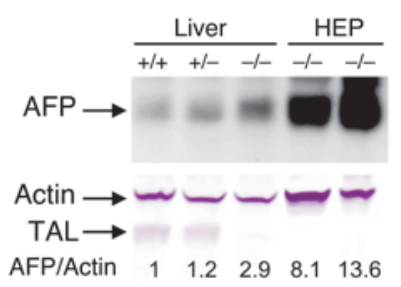

$\begin{array}{llllll}\text { AFP/Actin } \quad 1 & 1.2 & 2.9 & 8.1 & 13.6\end{array}$

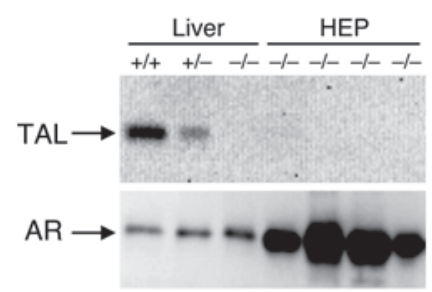

AR $1.0 \quad 0.91 .29 .521177 .8$
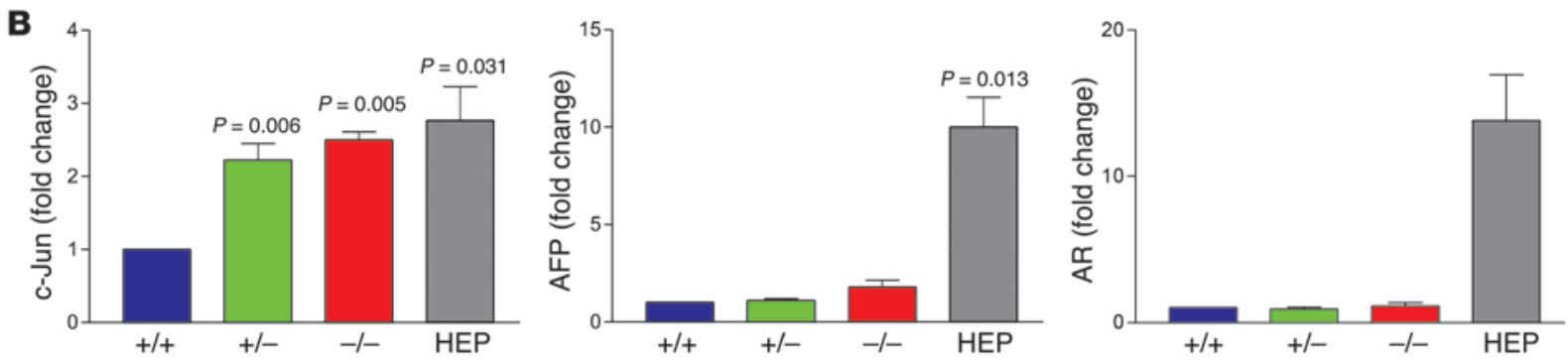

Figure 5

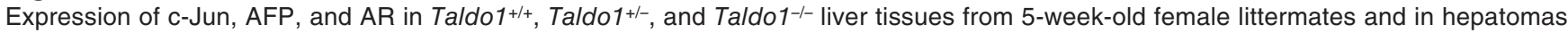
(HEP) from Taldo1 ${ }^{-1-}$ mice. While c-Jun and AFP were detected in whole cell lysates, AR was analyzed in cytosolic extracts (73). (A) Western blot detection of c-Jun, AFP, and AR expression relative to $\beta$-actin in livers and hepatoma tissues. Numbers below blots indicate densitometry values, which were normalized to those of Taldo1+/+ livers, set at 1.0. (B) Cumulative analyses of c-Jun/actin, AFP/actin, and AR/actin levels by

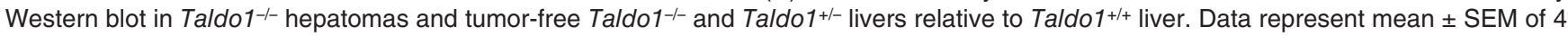
littermate sets and 4 hepatomas. $P$ values denoting significant differences versus Taldo ${ }^{+/+}$mice are shown. 
A

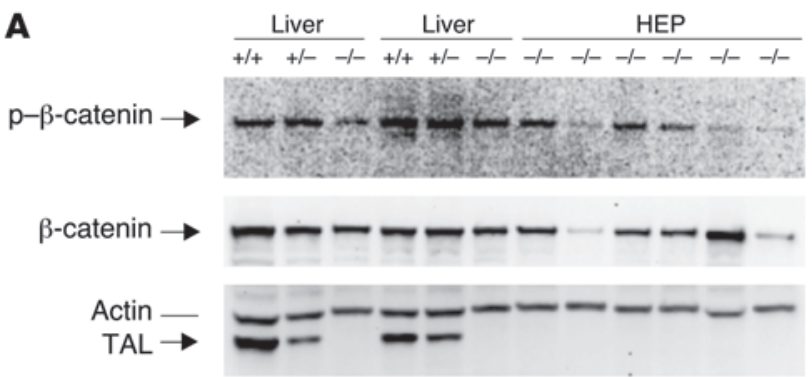

$\begin{array}{lllllllllllll}p-\beta \text {-catenin/ } \beta \text {-catenin } & 1 & 0.8 & 0.3 & 1 & 0.9 & 0.6 & 0.5 & 0.4 & 0.5 & 0.3 & 0.1 & 0.3\end{array}$

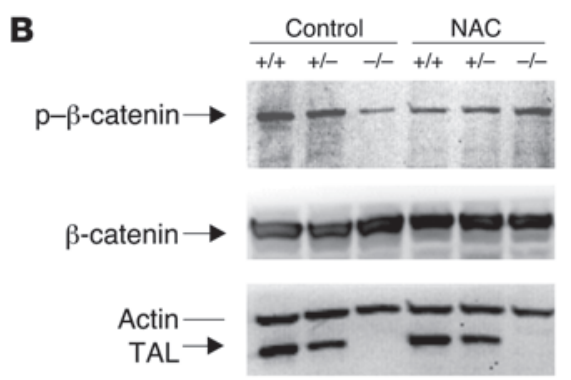

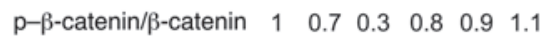

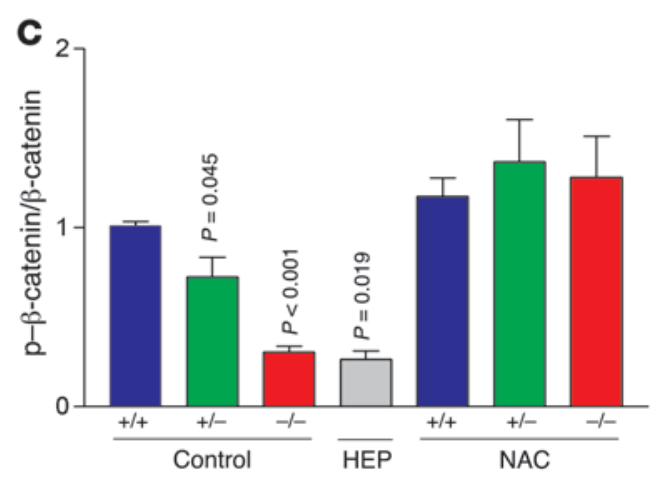

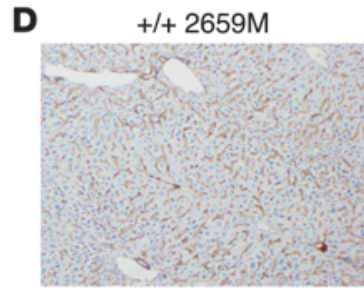

$-1-735 \mathrm{M} \mathrm{HCC}$

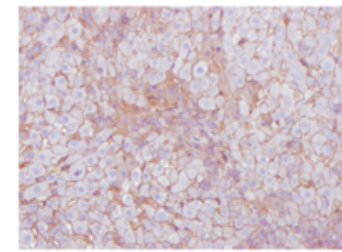

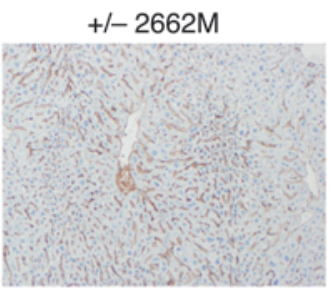

$-1-464 \mathrm{~F} \mathrm{HCC}$

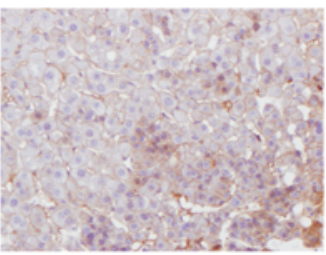

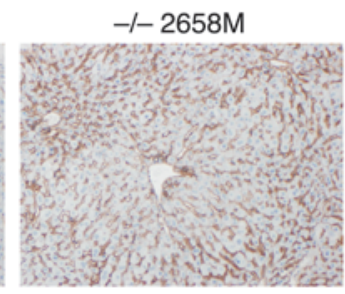

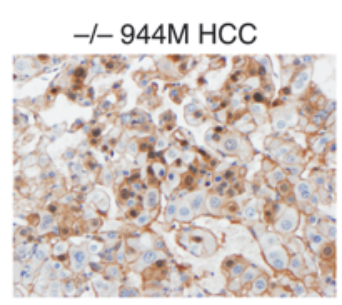

\section{Figure 6}

Effect of TAL deficiency on phosphorylation and intracellular localization of $\beta$-catenin in Taldo $1^{+/+}$, Taldo $1^{+/-}$, and Taldo $1^{-/-}$liver tissues from 10- to 12-week-old littermates and hepatomas from Taldo1-1- mice. (A) Western blot detection of phosphorylated $\beta$-catenin (p- $\beta$-catenin) relative to $\beta$-catenin in liver and hepatoma tissues. As a loading control, $p-\beta$-catenin and $\beta$-catenin levels were normalized to actin for each sample. (B) Effect of lifelong NAC treatment on $p-\beta$-catenin/ $\beta$-catenin levels in liver tissues of 12 -week-old littermates. Numbers below blots show $p-\beta$-catenin/ $\beta$-catenin ratios (i.e., [p- $\beta$-catenin/actin] $/\left[\beta\right.$-catenin/actin]), which were normalized to those of Taldo $1^{+/+}$livers, set at 1.0 . (C) Cumulative analyses of $p-\beta$-catenin/ $\beta$-catenin levels in liver tissues of 12 -week-old littermates, hepatomas, and liver tissues from NAC-treated 10 - to 12 -week-old littermates. Data represent mean \pm SEM of 4 littermate sets and 13 hepatomas. $P$ values denoting significant differences versus control-treated Taldo1+/+ mice are shown. (D) Immunohistochemical analysis of $\beta$-catenin expression in liver tissues of 12-week-old littermates and in Taldo $1^{-1-}$ livers with HCC. Original magnification, $\times 100$.

recognized as an essential signaling mechanism involved in both development and disease processes of the liver, including metabolism, oxidative stress, zonation, hepatitis, and hepatocarcinogenesis $(33,34)$. Moreover, $\beta$-catenin is the main effector of the canonical Wnt signaling pathway (34). Normally, $\beta$-catenin is located in the cytosol and it is associated with E-cadherin near the cell membrane. Turnover of $\beta$-catenin is dependent on phosphorylation by glycogen synthase kinase- $3 \beta$ (GSK3 $\beta$ ), which forms a degradation complex with the scaffolding proteins APC, Axin 1, and Axin 2 (33). Increased signaling through Wnt/Frizzled leads to inactivation of GSK3 $\beta$ that limits the phosphorylation of $\beta$-catenin. Unphosphorylated $\beta$-catenin is protected from ubiquitination and proteasomal degradation and accumulates in the cytoplasm, which is followed by nuclear translocation (34) and activation of $\mathrm{T}$ cell factor and various target genes, such as c-Jun (35). Oxidative stress enhances the transcriptional coactivator function of $\beta$-catenin (36). Therefore, we examined $\beta$-catenin expression in the livers of 4-month-old Taldo $1^{+/+}$, Taldo $1^{+/}$, and Taldo $1^{-/-}$littermates and in 13 hepatomas. As shown in Figure 6, A-C, phosphorylation of $\beta$-catenin was reduced in Taldo $1^{-/-}$livers and hepatomas, and to a lesser extent in Taldo $1^{+/-}$livers, relative to Taldo $1^{+/+}$litter- mates. The expression of $\beta$-catenin was increased in the perivenous region, consistent with its role in zonal regulation of liver physiology (34). This expression was confined to the cytosol adjacent to the plasma membrane in tumor-free livers (Figure 6D). Conversely, $\beta$-catenin showed diffuse accumulation in the cytosol of 13 of 13 Taldo $1^{-/}$hepatomas and translocation into the nuclei of tumor cells in 4 of 13 Taldo1 $1^{-/-}$hepatomas (Figure 6D). Western blot analysis showed similar protein levels of p21 (also designated WAF1/Cip1) in 8-week-old Taldo $1^{+/+}$, Taldo $1^{+/}$, and Taldo1 $1^{-/-}$livers (data not shown).

Resistance of Taldo1 $1^{-/}$bepatocytes to Fas-dependent apoptosis. Growing evidence supports the involvement of CD95/Fas cell death receptor-initiated apoptosis in physiological regulation of hepatocyte turnover (8). The role of GSH in Fas-induced liver cell death is the subject of controversy. Depletion of GSH, which is required for activity of caspases, can abrogate Fas-dependent apoptosis of the liver (37). Alternatively, prolonged GSH depletion may enhance Fasinduced apoptosis (38). Upon in vitro treatment with Jo2 anti-Fas antibody, caspase 3 activity was reduced in Taldo $1^{-/}$and Taldo $1^{+/-}$ hepatocytes compared with Taldo $1^{+/+}$controls (Figure 7A). After i.p. injection of Jo2 antibody, survival of Taldo1 $1^{-/}$mice was markedly 
A

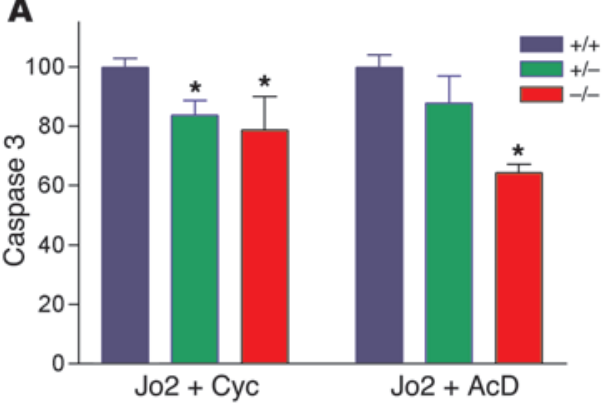

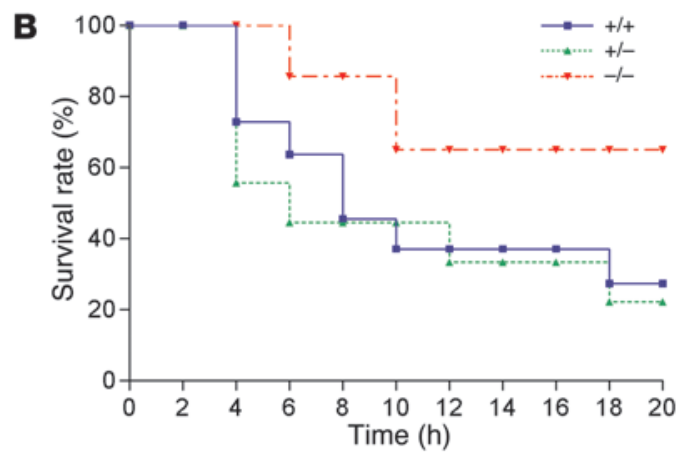

$+1-$
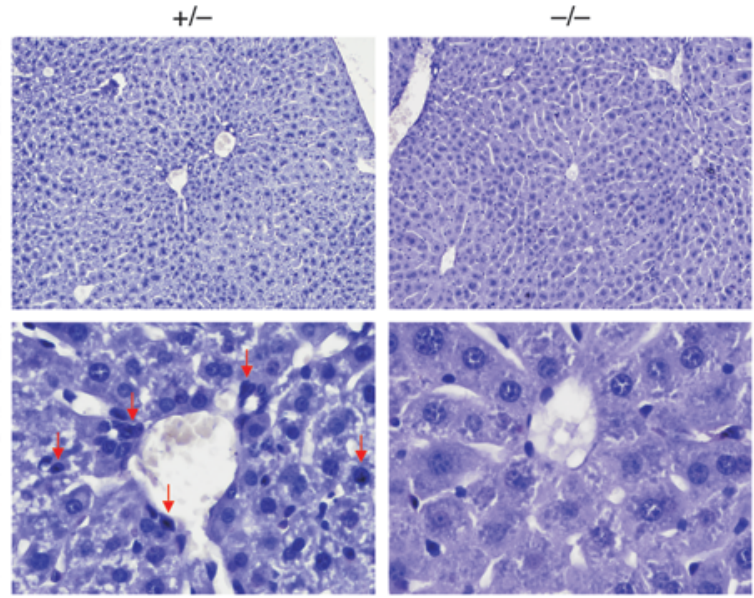

\section{Figure 7}

Resistance of Taldo1-/- hepatocytes to Fas-induced apoptosis. (A) Fas-induced apoptosis of hepatocytes in vitro by activation of caspase 3. Hepatocytes were incubated as described in Methods with anti-Fas antibody (Jo2, $0.5 \mu \mathrm{g} / \mathrm{ml}$ ) and $50 \mu \mathrm{g} / \mathrm{ml} \mathrm{cycloheximide} \mathrm{(Cyc)} \mathrm{or} 50 \mathrm{ng} / \mathrm{ml}$ actinomycin $D(A c D)$. Mean \pm SEM values from 4 litters per independent experiment are expressed relative to hepatocytes from Taldo $1^{+/+}$littermates, set as $100 \%$. ${ }^{*} P<0.05$ versus Taldo $1^{+/+}$. (B) Survival of 8 - to 10 -week-old Taldo $1^{+/+}(n=22)$, Taldo $1^{+/-}(n=15)$, and Taldo $1^{-/-}$littermate mice $(n=14)$ following i.p. injection with Jo2 antibody (10 $\mu \mathrm{g} / 30 \mathrm{~g}$ body weight). Log-rank test showed increased survival of Taldo ${ }^{-1-}$ mice

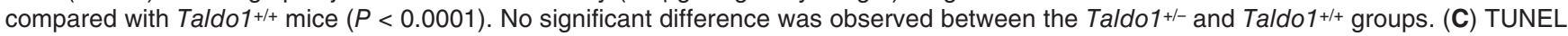
staining of liver sections 4 hours after i.p. injection with Jo2 antibody. Condensed and fragmented apoptotic nuclei (red arrows) in Taldo $1^{+/+}$and Taldo $1^{+/}$livers were visualized by staining with Vector Black substrate. Sections were counterstained with hematoxylin. Original magnification, $\times 100$ (top row); $\times 400$ (bottom row).

increased relative to Taldo $1^{+/+}$or Taldo $1^{+/-}$mice $(P<0.001$, log-rank test; Figure 7B). Liver sections obtained 4 hours after Jo2 injection showed significantly fewer TUNEL-positive apoptotic nuclei in Taldo $1^{-/-}$hepatocytes $(11 \% \pm 4.5 \%)$ relative to Taldo $1^{+/+}(79 \% \pm 15 \%$; $P=0.016)$ or Taldo1 ${ }^{+/-}$controls $(49 \% \pm 8.5 \% ; P=0.034$; Figure $7 \mathrm{C})$.

Increased susceptibility of Taldo $1^{-1-}$ mice to APAP-induced liver failure. APAP overdose is the leading cause of acute liver failure in the US $(13,39)$. APAP is converted by hepatic cytochrome P450 enzymes, primarily CYP2E1, to the toxic intermediate compound NAPQI, which is rapidly detoxified by GSH (40). To examine the ability of Taldo $1^{-/-}$mice to resist APAP-induced oxidative stress, 10 -weekold Taldo $1^{+/-}$, Taldo $1^{-/-}$, and Taldo $1^{+/+}$littermates were injected with $100-800 \mathrm{mg} / \mathrm{kg}$ APAP i.p. Upon injection with $800 \mathrm{mg} / \mathrm{kg}$ APAP, Taldo1 $1^{-/}$mice had increased mortality (Figure $8 \mathrm{~A}$ ) as a result of fulminant liver necrosis (Figure 8B). After 24 hours, 8 of 23 (35\%) Taldo1 $1^{-/-}$mice survived, while 17 of 28 (61\%) Taldo1 ${ }^{+/-}$and 16 of $26(62 \%)$ Taldo $1^{+/+}$mice survived $(P=0.027$; Figure 8 A). CYP2E1 protein levels were similar in the livers of Taldo $1^{+/+}$(normalized at $1.0 \pm 0.05)$, Taldo1 ${ }^{+/-}(1.2 \pm 0.3 ; P=0.33)$, and Taldo1 $1^{-/}(1.0 \pm 0.1$; $P=0.95)$ littermates. Likewise, CYP1A2 protein levels were similar in the livers of Taldo $1^{+/+}$(normalized at $\left.1.0 \pm 0.02\right)$, Taldo $1^{+/-}(1.1 \pm 0.2$; $P=0.84)$, and Taldo $1^{-/-}(0.9 \pm 0.3 ; P=0.79)$ littermates (Supplemental Figure 5). Thus, TAL deficiency did not influence expression of CYP2E1 or CYP1A2, which suggests that increased susceptibility to APAP was not related to changes in expression of these genes.

At 1 hour after APAP treatment, an interval insufficient to produce observable histological changes, increased phosphorylation of 46- and 54-kDa JNK (Figure 8C) and increased kinase activity of JNK were noted in Taldo1 $1^{-/}$livers (Figure 8D). Neither TAL deficiency nor APAP treatment influenced JNK protein levels (Figure $8 \mathrm{C}$ ). We investigated the impact of the JNK inhibitor SP600125 (41) on APAP-induced activation of the JNK pathway. Although pretreatment with $30 \mathrm{mg} / \mathrm{kg} \mathrm{SP} 600125$ caused a greater than 5 -fold reduction of JNK activation (Figure 8E), it failed to improve the overall survival of Taldo1 $1^{-1-}$ mice (Supplemental Figure 6).

Lifelong administration of NAC prevents APAP-induced liver failure, reverses Fas resistance, and blocks hepatocarcinogenesis in Taldo $1^{-1-}$ mice. NAC, a precursor of de novo GSH biosynthesis, is a potent antioxidant and the treatment of choice for APAP-induced liver failure (42). In addition to enhancing APAP toxicity, ongoing chronic oxi- 
A

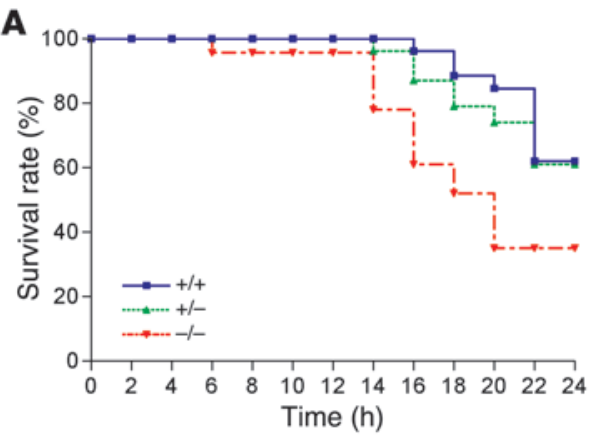

C
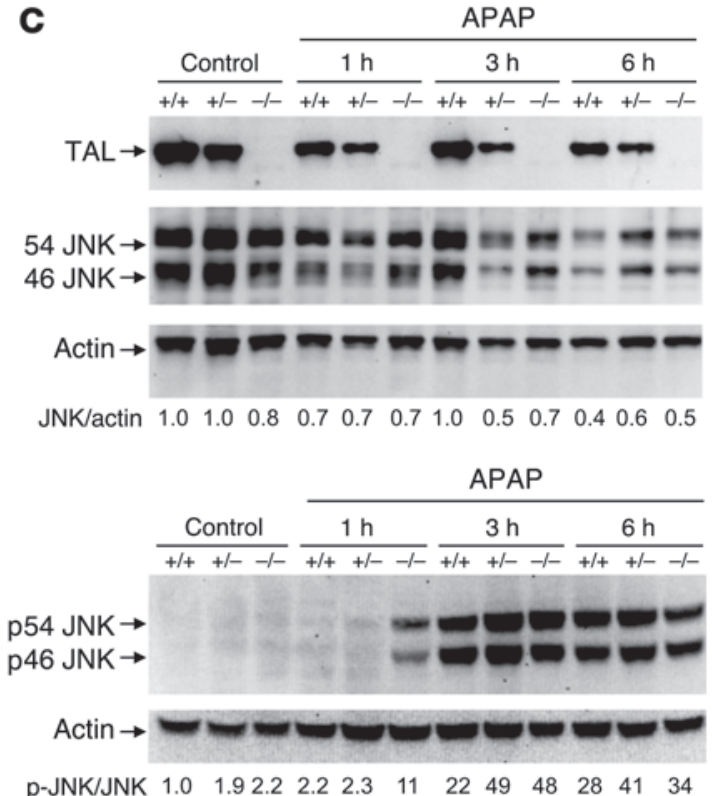

B
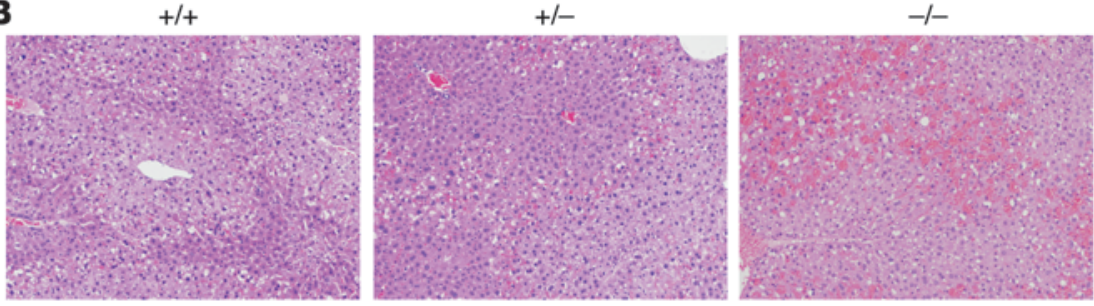

D
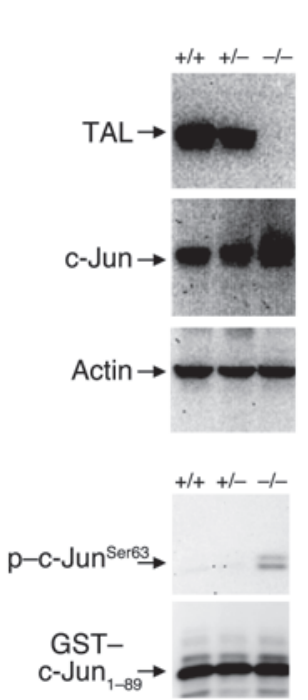

E

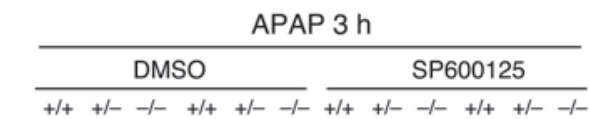

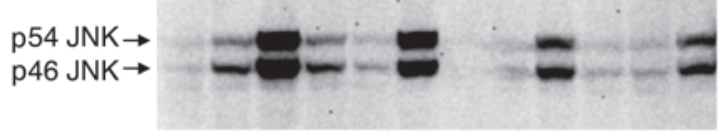
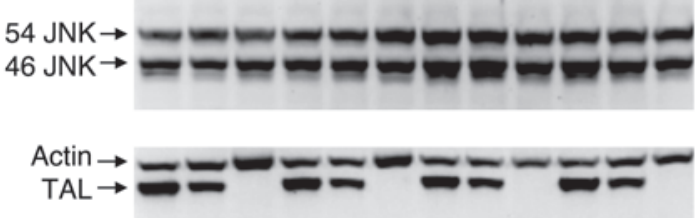

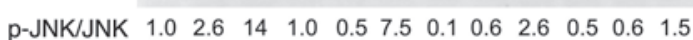

\section{Figure 8}

Increased susceptibility of Taldo $1^{-/-}$mice to liver failure induced by APAP. (A) Survival of Taldo1 $1^{+/+}(n=26)$, Taldo $1^{+/-}(n=28)$, and Taldo $1^{-/-}$littermates $(n=23)$ injected with $800 \mathrm{mg} / \mathrm{kg}$ APAP. Log-rank test showed reduced survival of Taldo $1^{-/-}$compared with $T$ aldo $1^{+/+}$mice $(P=0.027)$. No significant difference was observed between the Taldo $1^{+/}$and Taldo $1^{+/+}$groups. (B) H\&E-stained liver sections obtained 6 hours after APAP injection. Hemorrhagic necrosis, characterized by hepatocyte vacuolization and extravasation of erythrocytes, was enhanced in Taldo $1^{-1-}$ liver. Original magnification, $\times 100$. (C) Western blot detection of 46 - and $54-k D a$ JNK and their state of phosphorylation (p46 and p54 JNK) in APAPinjected and untreated control mice. Numbers below blots show $\mathrm{p}-\mathrm{JNK} / \mathrm{JNK}$ levels, which were determined relative to actin and normalized to untreated Taldo1+/+ protein lysates, set as 1.0. (D) Assessment of JNK activity by in vitro phosphorylation of GST-C-Jun ${ }_{1-89}$ fusion protein in APAP-treated liver. TAL, C-Jun, and actin levels were detected by Western blot of liver cell lysates. In vitro phosphorylation of GST-C-Jun was detected by Western blot analysis using anti-phospho-C-Jun ${ }^{\text {Ser63 }}$ antibody. (E) Effect of SP600125 on APAP-induced activation of JNK. Littermate 12-week-old mice were pretreated with SP600125 or DMSO control as described in Methods 1 hour prior to APAP exposure. Numbers below blots show $\mathrm{p}$-JNK/JNK levels 3 hours after APAP treatment; values were determined relative to actin and normalized to untreated Taldo ${ }^{+/+}$ protein lysates, set as 1.0 .

dative stress may also contribute to hepatocarcinogenesis through dephosphorylation of $\beta$-catenin, activation of c-Jun, and inhibition of Fas apoptosis. Therefore, we introduced $10 \mathrm{~g} / 1 \mathrm{NAC}$ to the drinking water of Taldo $1^{+/}$breeding pairs and their offspring throughout their lives. Such intervention for approximately 3 months increased the GSH content of Taldo1 $1^{--}$livers by $60 \%$, thereby compensating for GSH diminution. In addition to GSH content (measured by both HPLC and flow cytometry), NADPH levels (measured by HPLC), ROI levels (measured by H\&E fluorescence), NO production (measured by DAF-FM fluorescence), and mitochondrial mass (measured by MitoTracker Green fluorescence) were normalized in hepatocytes isolated from 4 NAC-treated Taldo $1^{-/-}$mice rela- tive to those from 4 NAC-treated Taldo $1^{+/+}$littermates. NAC treatment also normalized the number of PCNA- and TUNEL-positive nuclei in Taldo1 $1^{-/}$livers (data not shown). Each animal in 4 sets of 12-week-old Taldo $1^{+/+}(n=5)$, Taldo1 ${ }^{+/-}(n=5)$, and Taldo1 ${ }^{-/-}$littermates $(n=4)$ given lifelong NAC survived i.p. challenge with 800 $\mathrm{mg} / \mathrm{kg}$ APAP. Strikingly, NAC treatment restored susceptibility to Fas-induced cell death in Taldo1 $1^{-/}$mice (Supplemental Figure 7). NAC treatment also normalized $\beta$-catenin phosphorylation (Figure 6, B and C) and c-Jun expression in Taldo $1^{-/-}$livers (data not shown). To investigate whether normalization of $\beta$-catenin phosphorylation and c-Jun expression and restoration of Fas apoptosis by NAC also prevented hepatocarcinogenesis, mice were kept alive 


\section{Table 2}

Effect of NAC administration on HCC development

\begin{tabular}{lccc} 
HCC frequency & No NAC & NAC & $P^{A}$ \\
Taldo1+/+ & $1 / 101$ & $0 / 19$ & 1.000 \\
Taldo1+- & $39 / 190$ & $0 / 43$ & 0.0014 \\
Taldo1-/- & $45 / 52$ & $0 / 17$ & $<0.0001$ \\
\hline
\end{tabular}

Values denote ratio of HCC presence/absence. NAC was administered at $10 \mathrm{~g} / \mathrm{l}$ in drinking water. Mice were sacrificed at approximately 86 weeks. ${ }^{A}$ NAC versus untreated. $P$ values were determined by Fisher exact test.

up to 86 weeks, well beyond the mean age of death due to HCC (74 weeks). Entire litters were sacrificed together for macroscopic and microscopic analysis of the liver. Of the NAC-treated litters, none

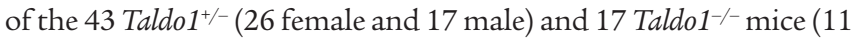
female and 6 male) developed hepatoma (Table 2). Lipid accumulation persisted after NAC treatment (Supplemental Figure 8), which suggests that a metabolic defect such as stimulation of lipogenesis by X5P (43), rather than oxidative stress, was responsible for steatosis in Taldo $1^{-/-}$livers.

\section{Discussion}

The present data identify TAL deficiency as a cause of hepatocarcinogenesis. Tumor development in the liver is dependent on a multistep process of genetic changes reflecting adaptation to oxidative stress $(27,29)$, with prominent involvement of c-Jun (11, 44). A redox-sensitive transcription factor, c-Jun inhibits liver cell death, promotes hepatocyte proliferation $(12,30)$, and stimulates expression of AFP (31), a biomarker of HCC development in cirrhotic patients (4). We found that HCC was associated with overexpression of AFP and led to a moribund state in Taldo1 $1^{-/-}$mice at the mean age of 74 weeks, in contrast to the expected lifespan of 130 weeks (45). The mean age of hepatoma presentation in humans is 61.8 years (46). Thus, TAL deficiency is a relevant model of hepatocarcinogenesis in men. Paradoxically, activation of the JNK/c-Jun pathway also promotes oxidative stress-induced acute liver cell injury, such as that triggered by APAP (47). Although JNK activation was accelerated in Taldo1 $1^{-/}$mice, inhibition of JNK failed to improve their overall survival. Levels of LPO, MDA, and 4-HNE adducted proteins increased in Taldo1 ${ }^{-/-}$livers (Supplemental Figure 4), which suggests a role for preexisting damage from oxidative stress and increased vulnerability of Taldo $1^{-/-}$mice. Indeed, supplementation of NAC to the drinking water of Taldo $1^{+/}$breeding pairs and their offspring throughout their lives not only increased GSH and NADPH levels, but also reduced LPO, MDA, and 4-HNE production (Supplemental Figure 4) and completely prevented the APAP-induced death of Taldo1 $1^{-/-}$mice. Thus, TAL deficiencyinduced oxidative stress predisposes to both hepatocarcinogenesis and APAP-induced fulminant liver failure.

Hepatocarcinogenesis occurs in the context of NAFLD and NASH. Mutations in the gene encoding TAL have been associated with liver disease in children from 3 families $(15,17,48)$, but have not been documented in liver cancer. Homozygous deletion of $3 \mathrm{bp}$ (coding for Ser171) of the TALDO1 gene was described in a 9-year-old girl with liver cirrhosis (15). Deletion of Ser171 caused misfolding, proteasome-mediated degradation, and complete deficiency of TAL in fibroblasts and lymphoblasts of this patient (49). A second TALDO1deficient patient $(\mathrm{R} 192 \mathrm{H})$ died at the age of 18 days from liver fail- ure, cardiomyopathy, and respiratory failure (16). This patient also had Turner syndrome mosaicism $(\mathrm{XX} / \mathrm{XO})$ contributing to the phenotype (16). An additional 4 patients with deletion of Ser171 in TALDO1 developed multiorgan disease, including liver steatosis, cirrhosis of varying severity, and genitourinary malformations (17). Because all children with TAL deficiency were offspring of consanguineous parents, mutations in genes other than TALDO1 may have influenced the clinical presentation. The phenotype of mice with a targeted disruption of Taldo1 provides direct evidence for a protective role of this gene against the development of liver disease.

The metabolic basis of liver disease is characterized by the accumulation of S7P, R5P, X5P, and C5-polyols and by depletion of $\mathrm{NADPH}$ and GSH, which underlie mitochondrial dysfunction and oxidative stress. The accumulation of 5-carbon sugar phosphates $\mathrm{X} 5 \mathrm{P}$ and R5P (50) as well as ADP and ATP inhibited the activities of G6PD and 6-phosphogluconate dehydrogenase (51) and the production of NADPH (Supplemental Figure 9). Oxidative stress was attributed to GSH diminution exceeding the reduction in mitochondrial ROI production. Diminished G6PD was accompanied by increased SOD, CAT, and GPx activities in Taldo1 ${ }^{-1-}$ livers and increased CAT and GPx activities in Taldo1 ${ }^{+/-}$livers (Supplemental Table 1), thus reflecting protective measures against oxidative stress. By microarray analysis, expression of GST genes was unchanged (alpha 2, GST alpha 4, mu 6, zeta 1, microsomal GST 1, microsomal GST 3, omega 1, pi 2, theta 1, and theta 2) or increased less than 2-fold (mu 2, 1.8-fold; mu 3, 1.3-fold) in Taldo1-/- livers compared with littermate controls (data not shown). The impact of TAL deficiency extended beyond the consequences of GSH deficit. Reduction of liver GSH by as much as $75 \%$ of baseline has not previously been associated with spontaneous liver disease, cirrhosis, or hepatomas $(52,53)$. Thus, we conclude that GSH depletion is not responsible for hepatocarcinogenesis in Taldo1 $1^{-/}$mice. The restoration of GSH in NAC-treated mice was accompanied by the normalization of NAPDH, LPO, MDA, and 4-HNE levels and of the number of PCNA- and TUNEL-positive nuclei in Taldo1 $1^{-/-}$and Taldo $^{+/-}$livers, which suggests that lifelong supplementation of NAC has a sparing effect on the use of NADPH and works as a potent antioxidant. Of note, GSH-depleted livers of mice deficient in gamma-glutamyltranspeptidase were deprived of fat (53). Conversely, we observed an increase in fat deposition in Taldo1-/livers. GSH normalization by NAC treatment failed to prevent steatosis, which suggests that lipid deposition was independent of oxidative stress. Steatosis is likely to happen through stimulation of lipogenesis by X5P (43). C5-polyols also accumulated in Taldo1 $1^{--}$urine and liver. C5-sugars are metabolized to C5-polyols by NADPH-dependent AR (54), which further depleted NADPH (Supplemental Figure 9). Moreover, JNK activity and c-Jun expression are stimulated not only by oxidative stress $(12,30)$, but also by hyperosmolarity, such as accumulation of sugars and polyols (55-58). In turn, JNK and c-Jun inhibit Fas apoptosis (59). Thus, the accumulation of C5-sugars and C5-polyols may be a unique factor contributing to liver disease in TAL deficiency. Diminished phosphorylation of $\beta$-catenin, a known activator of c-Jun expression (35), was associated with diffuse accumulation of $\beta$-catenin in the cytosol of Taldo1 $1^{-/}$hepatomas and translocation of $\beta$-catenin into the nuclei of tumor cells. Phosphorylation of $\beta$-catenin was normalized in the livers of NAC-treated mice, which may be an important factor in preventing hepatocarcinogenesis in Taldo $1^{-/}$mice. Activation of $\beta$-catenin has previously been demonstrated in up to $90 \%$ of human HCC cases $(34,60,61)$, further 
supporting the notion that TAL deficiency is a relevant model for human hepatocarcinogenesis.

Inhibited apoptosis allows the survival of hepatocytes with genetic mutations that provide growth advantage in the context of oxidative stress $(8,10,62)$. Depletion of GSH, which is required for activity of caspases, can abrogate Fas-dependent apoptosis of the liver (37). Alternatively, ATP concentration is key factor in the decision between apoptosis and necrosis; as depletion of ATP shifts susceptibility from apoptosis to necrosis (63), the loss of $\Delta \psi_{\mathrm{m}}$ and reduced ATP/ADP ratio may thus contribute to inhibited apoptosis. However, production of NADPH, GSH, and NO were all corrected in NACfed mice, which suggests that these metabolic changes are located upstream of mitochondrial dysfunction in TAL deficiency.

In summary, these results provided insight into the mechanisms of oxidative stress in the liver by revealing a dominant role for the TAL-controlled nonoxidative PPP in the spontaneous development of NASH, cirrhosis, nodular hyperplasia, and HCC and by identifying TAL as a tumor suppressor in the liver. The metabolic basis of oxidative stress was characterized by the accumulation of S7P and the failure to recycle R5P for the oxidative branch of the PPP, which resulted in diminution of NADPH and GSH (Supplemental Figure 9). Hepatocarcinogenesis in TAL deficiency appears to require diminished GSH and the resultant curtailment of $\beta$-catenin phosphorylation and Fas-dependent apoptosis. The success of NAC therapy in normalizing of GSH and preventing hepatocarcinogenesis in Taldo1 $1^{-/-}$mice suggests that a similar approach may benefit TAL-deficient patients.

\section{Methods}

Mice. Taldo $1^{-/-}$mice were developed by inactivation of the Taldo1 genomic locus (18). Taldo1 $1^{-/-}$male mice are infertile as a result of sperm dysmotility

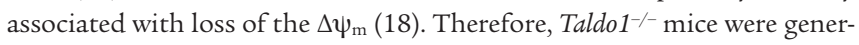
ated through breeding heterozygotes and genotyping by PCR and Western blot analysis of TAL expression in excised tail tissue (18). All studies in animals were approved by the Institutional Committee for the Humane Use of Animals of the State University of New York Upstate Medical University College of Medicine.

In vivo treatments. Fas-induced apoptosis was provoked by i.p. injection of $10 \mu \mathrm{g} / 30 \mathrm{~g}$ body weight of Fas-specific Jo2 hamster monoclonal antibody (catalog no. 554254; BD Biosciences - Pharmingen) in $100 \mu \mathrm{l}$ PBS. APAP was injected i.p. at a dose range of $100-800 \mathrm{mg} / \mathrm{kg}$. Fas- or APAP-treated mice were continuously monitored and euthanized at predetermined time points or at the earliest sign of distress. JNK inhibitor SP600125 (catalog no. 420119; Calbiochem/EMD Biosciences) was resuspended in 10\% DMSO and 90\% PBS and administered at doses of $10-30 \mathrm{mg} / \mathrm{kg}$ i.p. 1 hour prior to APAP injection. When testing the effect of SP600125 on JNK activation, control mice were injected with an equal volume of $10 \%$ DMSO and $90 \%$ PBS. NAC (10 mg/ml, pH 6.7; Sigma-Aldrich) was administered in the drinking water. To test glucose tolerance, after fasting overnight $(\sim 14$ hours), mice were injected i.p. with sterile-filtered glucose $(2 \mathrm{~g} / \mathrm{kg}$, from a solution of $10 \%$ glucose and $0.9 \% \mathrm{NaCl}$ ). Tail vein blood was obtained by needle pricks before and 15, 30, 60, and 120 minutes after glucose injection. Glucose levels were assessed using a OneTouch Ultra electronic blood glucose monitor (LifeScan), as described previously (64).

Liver pathology. Paraffin-embedded sections were stained with H\&E and evaluated blindly as to the genotype for dysplasia, cirrhosis, and HCC using previously established criteria $(19,65)$. Clearly circumscribed nodular lesions with dysplastic cells and small or large cell change were regarded as dysplastic nodules. Only lesions with definitive invasion, manifested by irregular and infiltrative interface into the adjacent liver, were regarded as carcinoma. No adenomas were observed. Fat-storing hepatic stellate or Ito cells were identified by staining with antibody to glial fibrillary acidic protein (66). Fat deposition and fibrosis were detected by Oil-Red-O and Klatskin trichrome staining $(67,68)$, respectively. Apoptosis of liver cells of Fas antibody-injected animals was assessed by TUNEL staining using the In Situ Cell Death Detection kit from Roche. Paraffin-embedded liver sections were deparaffinized, rehydrated, and labeled with fluorescein-dUTP using terminal deoxynucleotidyl transferase (TdT). During this incubation period, TdT catalyzes the addition of fluorescein-dUTP at free $3^{\prime}-\mathrm{OH}$ groups in single- and double-stranded DNA breaks. After washing, the label incorporated at the damaged sites of the DNA was marked by an anti-fluorescein antibody conjugated with the reporter enzyme alkaline phosphatase (AP). After washing to remove unbound enzyme conjugate, the AP retained in the immune complex at the site of the damaged DNA was visualized by the addition of Vector Black AP Substrate (Vector Labs).

Electron microscopy. Freshly excised tissues were fixed overnight in PBS with $2.5 \%$ glutaraldehyde, postfixed in $1 \% \mathrm{OsO}_{4}$, dehydrated in graded ethanol series, infiltrated with propylene oxide, and embedded in Araldite 502 epoxy resin (Electron Microscopy Sciences). Ultrathin sections were stained with uranyl acetate and Reynold's lead citrate prior to examination with a Tecnai BioTWIN 12 transmission electron microscope (FEI).

Isolation and in vitro culture of mouse hepatocytes. Livers of 8- to 10-week-old Taldo $1^{+/+}$, Taldo1 $1^{+/}$, and Taldo1 $1^{-/-}$female littermates were cannulated through the portal vein, and, after nicking the inferior vena cava, perfused by a 2 -step procedure using $\mathrm{Ca}^{2+}$-free preperfusion buffer and perfusion medium with $0.02 \%$ type IV collagenase and $0.4 \mathrm{U} / \mathrm{ml}$ elastase (both from Sigma-Aldrich), as previously described (69). Isolated hepatocytes with viability greater than $90 \%$ were resuspended in DMEM containing $100 \mathrm{IU} /$ $\mathrm{ml}$ penicillin $\mathrm{G}, 100 \mu \mathrm{g} / \mathrm{ml}$ streptomycin, $0.2 \mathrm{mg} / \mathrm{ml}$ serum bovine albumin, $1 \mathrm{mM}$ sodium pyruvate, and $10 \%$ fetal calf serum and kept on ice until staining for flow cytometry. For in vitro culture, $2.4 \times 10^{6}$ hepatocytes were plated in $60 \mathrm{~mm}$ Petri dishes precoated with type I collagen (catalog no. C9791; Sigma-Aldrich). After 3 hours of incubation, plates were washed and incubated for 6 hours with fresh DMEM and used for in vitro nitrite assays using the Griess kit (Invitrogen). For Fas-induced apoptosis, $2.4 \times 10^{6}$ hepatocytes were plated in collagen-coated 24 -well plates. After 3 hours of incubation, plates were washed and incubated for 8 hours with $0.5 \mu \mathrm{g} / \mathrm{ml} \mathrm{Jo} 2$ in combination with $50 \mu \mathrm{g} / \mathrm{ml}$ cycloheximide or $50 \mathrm{ng} / \mathrm{ml}$ actinomycin D (8). Control cultures were incubated with DMEM, cycloheximide, or actinomycin D only. Caspase 3 activity of cell supernatants was measured by the DEVD-AFC cleavage assay (70).

Flow cytometric analysis of $\Delta \psi_{m}$, mitochondrial mass, NO and ROI production, GSH, and cytoplasmic and mitochondrial $\mathrm{Ca}^{2+}$ levels. Hepatocytes were analyzed on a BD LSRII flow cytometer equipped with $20 \mathrm{~mW}$ solid-state Nd-YAG (emission at $355 \mathrm{~nm}$ ), $20 \mathrm{~mW}$ argon (emission at $488 \mathrm{~nm}$ ), and $16 \mathrm{~mW}$ helium-neon lasers (emission at $634 \mathrm{~nm}$ ), as described in Supplemental Methods.

Western blot analysis. Protein lysates of freshly excised tissue were prepared by sonication using a sonic dismembrator (model 100; Fisher Scientific) in $300 \mu \mathrm{l}$ of buffer containing $20 \mathrm{mM}$ Tris- $\mathrm{HCl}$ (pH 7.5), $150 \mathrm{mM} \mathrm{NaCl}$, $1 \mathrm{mM} \mathrm{Na}{ }_{2}$ EDTA, $1 \mathrm{mM}$ EGTA, 1\% Triton, $2.5 \mathrm{mM}$ sodium pyrophosphate, $1 \mathrm{mM} \beta$-glycerophosphate, $1 \mathrm{mM} \mathrm{Na} \mathrm{VO}_{4}$, and $1 \mu \mathrm{g} / \mathrm{ml}$ leupeptin. Protein concentrations were determined by the Bradford method using the Bio-Rad Protein Assay (Bio-Rad). Protein lysates (40 $\mu \mathrm{g}$ unless otherwise indicated) were separated on a $12 \%$ SDS-polyacrylamide gel, electroblotted to nitrocellulose, and developed with specific antibodies as described in Supplemental Methods.

JNK assay. Liver tissue aliquots $(\sim 100 \mathrm{mg})$ were homogenized by sonication in $400 \mu \mathrm{l}$ of lysis buffer containing $20 \mathrm{mM}$ Tris (pH 7.4), $150 \mathrm{mM}$ $\mathrm{NaCl}, 1 \mathrm{mM}$ EDTA, $1 \mathrm{mM}$ EGTA, 1\% Triton, $2.5 \mathrm{mM}$ sodium pyrophosphate, $1 \mathrm{mM} \beta$-glycerophosphate, $1 \mathrm{mM} \mathrm{Na}_{3} \mathrm{VO}_{4}, 1 \mu \mathrm{g} / \mathrm{ml}$ leupeptin, and 
$1 \mathrm{mM}$ phenylmethylsulfonyl fluoride. JNK activity was tested by mixing $200 \mu \mathrm{g}$ of liver lysate protein with agarose beads coupled to GST-c-Jun ${ }_{1-89}$ fusion protein overnight at $4{ }^{\circ} \mathrm{C}$, followed by washing twice in lysis buffer and incubating with $200 \mu \mathrm{M}$ ATP in $50 \mu \mathrm{l}$ of $25 \mathrm{mM}$ Tris ( $\mathrm{pH} 7.5$ ), $5 \mathrm{mM}$ $\beta$-glycerophosphate, $2 \mathrm{mM} \mathrm{DTT,} 0.1 \mathrm{mM} \mathrm{Na}_{3} \mathrm{VO}_{4}$, and $10 \mathrm{mM} \mathrm{MgCl}_{2}$ for 30 minutes at $30^{\circ} \mathrm{C}$. Phosphorylation of GST-c-Jun $1-89$ was detected by Western blot analysis using anti-phospho-c-Jun ${ }^{\text {Ser63 }}$ antibody (Cell Signaling).

PPP and related metabolic enzyme activity assays. Enzyme activities were determined as described in Supplemental Methods.

Measurement of phosphorylated sugars and nucleotides. All samples were analyzed after methanol/water extraction by LC-MS/MS (25). Sugar phosphates and nucleotides were detected in the same sample. NADH and NADPH were separately analyzed after extraction with $\mathrm{KOH}(25,71)$. This method allowed parallel detection of NADH $(m / z 665)$, NADP $(m / z 743)$, and NADPH $(m / z 744)$ by CapLC/Q-TOF detection (25). ATP levels were also determined using the luciferin-luciferase kit (Invitrogen).

Microarray of gene expression. RNA extraction, reverse transcription, hybridization to Mouse Expression Set 430A GeneChip, validation by real-time PCR, and analyses of gene expression are described in Supplemental Methods.
Statistics. Results of parametric data are expressed as mean \pm SEM. Differences between Taldo $1^{+/-}$, Taldo $1^{-/}$, and Taldo $1^{+/+}$mice were determined by 2 -tailed Student's $t$ test. Nonparametric data were analyzed with $\chi^{2}$ or Fisher exact test for fewer than 5 observations. Survival curves were analyzed with the log-rank test. $P$ values were calculated with Prism (version 3.02; GraphPad); a $P$ value less than 0.05 was considered significant.

\section{Acknowledgments}

This work was supported in part by NIH grant RO1 DK 49221, by the Central New York Community Foundation, and by the Children's Miracle Network.

Received for publication March 24, 2008, and accepted in revised form March 11, 2009.

Address correspondence to: Andras Perl, Department of Medicine, State University of New York, 750 East Adams Street, Syracuse, New York 13210, USA. Phone: (315) 464-4192; Fax: (315) 4644176; E-mail: perla@upstate.edu.
1. Ahn, J., and Flamm, S.L. 2004. Hepatocellular carcinoma. Dis. Mon. 50:556-573.

2. Stickel, F., Schuppan, D., Hahn, E.G., and Seitz, H.K. 2002. Cocarcinogenic effects of alcohol in hepatocarcinogenesis. Gut. 51:132-139.

3. El Serag, H.B. 2002. Hepatocellular carcinoma and hepatitis $\mathrm{C}$ in the United States. Hepatology. 36:S74-S83.

4. Nguyen, M.H., and Keeffe, E.B. 2002. Screening for hepatocellular carcinoma. J. Clin. Gastroenterol. 35:S86-S91.

5. Moradpour, D., and Blum, H.E. 2005. Pathogenesis of hepatocellular carcinoma. Eur. J. Gastroenterol. Hepatol. 17:477-483.

6. Eichhorst, S.T., et al. 2004. Suramin inhibits death receptor-induced apoptosis in vitro and fulminant apoptotic liver damage in mice. Nat. Med. 10:602-609.

7. Elmore, S.P., Qian, T., Grissom, S.F., and Lemasters, J.J. 2001. The mitochondrial permeability transition initiates autophagy in rat hepatocytes. FASEBJ. 15:2286-2287.

8. Ni, R., et al. 1994. Fas-mediated apoptosis in primary cultured mouse hepatocytes. Exp. Cell Res. 215:332-337.

9. Zou, C., et al. 2007. Lack of Fas antagonism by Met in human fatty liver disease. Nat. Med. 13:1078-1085.

10. Park, S.M., et al. 2008. CD95 signaling deficient mice with a wild-type hematopoietic system are prone to hepatic neoplasia. Apoptosis. 13:41-51.

11. Eferl, R., et al. 2003. Liver tumor development. c-Jun antagonizes the proapoptotic activity of p53. Cell. 112:181-92.

12. Hess, J., Angel, P., and Schorpp-Kistner, M. 2004. AP-1 subunits: quarrel and harmony among siblings. J. Cell Sci. 117:5965-5973.

13. Lee, W.M. 2004. Acetaminophen and the U.S. Acute Liver Failure Study Group: lowering the risks of hepatic failure. Hepatology. 40:6-9.

14. Jones, B.E., Liu, H., Lo, C.R., Koop, D.R., and Czaja, M.J. 2002. Cytochrome P450 2E1 expression induces hepatocyte resistance to cell death from oxidative stress. Antioxid. Redox Signal. 4:701-709.

15. Verhoeven, N.M., et al. 2001. Transaldolase deficiency: liver cirrhosis associated with a new inborn error in the pentose phosphate pathway. Am.J. Hum. Genet. 68:1086-1092.

16. Verhoeven, N.M., et al. 2005. A newborn with severe liver failure, cardiomyopathy and transaldolase deficiency. J. Inher. Metab. Dis. 28:169-179.

17. Valayannopoulos, V., et al. 2006. Transaldolase deficiency: A new cause of hydrops fetalis and neo- natal multi-organ disease. J. Pediatr. 149:713-717.

18. Perl, A., et al. 2006. Transaldolase is essential for maintenance of the mitochondrial transmembrane potential and fertility of spermatozoa. Proc. Natl. Acad. Sci. U. S. A. 103:14813-14818.

19. Ferrell, L. 2000. Liver pathology: cirrhosis, hepatitis, and primary liver tumors. Update and diagnostic problems. Mod. Pathol. 13:679-704.

20. Diehl, A.M. 2005. Lessons from animal models of NASH. Hepatol. Res. 33:138-144.

21. Sheth, S.G., Flamm, S.L., Gordon, F.D., and Chopra, S. 1998. AST/ALT ratio predicts cirrhosis in patients with chronic hepatitis $\mathrm{C}$ virus infection. Am. J. Gastroenterol. 93:44-48.

22. Angulo, P., et al. 2007. The NAFLD fibrosis score: a noninvasive system that identifies liver fibrosis in patients with NAFLD. Hepatology. 45:846-854.

23. Mayes, P.A. 1993. The pentose phosphate pathway \& other pathways of hexose metabolism. In Harper's biochemistry. R.K. Murray, D.K. Granner, P.A. Mayes, and V.W. Rodwell, editors. Appleton \& Lange. Norwalk, Connecticut, USA. 201-211.

24. Perl, A. 2007. The pathogenesis of transaldolase deficiency. IUBMB Life. 59:365-373.

25. Vas, G., et al. 2005. Study of transaldolase deficiency in urine samples by capillary LC-MS/MS. J. Mass Spectrom. 41:463-469.

26. Constantini, P., Chernyak, B.V., Petronilli, V., and Bernardi, P. 1996. Modulation of the mitochondrial permeability transition pore by pyridine nucleotides and dithiol oxidation at two separate sites. J. Biol.Chem. 271:6746-6751.

27. Hentze, H., Latta, M., Kunstle, G., Lucas, R., and Wendel, A. 2003. Redox control of hepatic cell death. Toxicol. Lett. 139:111-118.

28. Nisoli, E., et al. 2003. Mitochondrial biogenesis in mammals: the role of endogenous nitric oxide. Science. 299:896-899.

29. Wang, X.W., et al. 2002. Molecular pathogenesis of human hepatocellular carcinoma. Toxicology. 181-182:43-47.

30. Shaulian, E., and Karin, M. 2002. AP-1 as a regulator of cell life and death. Nat. Cell Biol. 4:E131-E136.

31. Houart, C., Szpirer, J., and Szpirer, C. 1992. C/EBP and c-JUN proteins activate the proximal enhancer of the developmentally regulated alpha-fetoprotein gene. Int. J. Dev. Biol. 36:109-114.

32. Cao, D., Fan, S.T., and Chung, S.S. 1998. Identification and characterization of a novel human aldose reductase-like gene. J. Biol. Chem. 273:11429-11435.

33. Fodde, R., and Brabletz, T. 2007. Wnt/beta-catenin signaling in cancer stemness and malignant behav- ior. Curr. Opin. Cell Biol. 19:150-158.

34. Thompson, M.D., and Monga, S.P. 2007. WNT/ beta-catenin signaling in liver health and disease. Hepatology. 45:1298-1305.

35. Anna, C.H., Iida, M., Sills, R.C., and Devereux, T.R. 2003. Expression of potential beta-catenin targets, cyclin D1, c-Jun, c-Myc, E-cadherin, and EGFR in chemically induced hepatocellular neoplasms from B6C3F1 mice. Toxicol. Appl. Pharmacol. 190:135-145.

36. Essers, M.A.G., et al. 2005. Functional interaction between beta-catenin and FOXO in oxidative stress signaling. Science. 308:1181-1184.

37. Hentze, H., Kunstle, G., Volbracht, C., Ertel, W., and Wendel, A. 1999. CD95-Mediated murine hepatic apoptosis requires an intact glutathione status. Hepatology. 30:177-185.

38. Haouzi, D., et al. 2001. Prolonged, but not acute, glutathione depletion promotes Fas-mediated mitochondrial permeability transition and apoptosis in mice. Hepatology. 33:1181-1188.

39. Lauterburg, B.H. 2002. Analgesics and glutathione. Am. J. Ther. 9:225-233.

40. Benson, G.D., Koff, R.S., and Tolman, K.G. 2005. The therapeutic use of acetaminophen in patients with liver disease. Am. J. Ther. 12:133-141.

41. Gunawan, B.K., et al. 2006. c-Jun N-terminal kinase plays a major role in murine acetaminophen hepatotoxicity. Gastroenterology. 131:165-178.

42. Gill, R.Q., and Sterling, R.K. 2001. Acute liver failure. J. Clin. Gastroenterol. 33:191-198.

43. Kabashima, T., Kawaguchi, T., Wadzinski, B.E., and Uyeda, K. 2003. Xylulose 5-phosphate mediates glucose-induced lipogenesis by xylulose 5-phosphate-activated protein phosphatase in rat liver. Proc. Natl. Acad. Sci. U. S. A. 100:5107-5112.

44. Jochum, W., Passegue, E., and Wagner, E.F. 2001. AP-1 in mouse development and tumorigenesis. Oncogene. 20:2401-2412.

45. Lee, C.K., Klopp, R.G., Weindruch, R., and Prolla, T.A. 1999. Gene expression profile of aging and its retardation by caloric restriction. Science. 285:1390-1393.

46. Hellerbrand, C., et al. 2001. Hepatocellular carcinoma in southern Germany: epidemiological and clinicopathological characteristics and risk factors. Dig. Dis. 19:345-351.

47. Czaja, M.J. 2007. Cell signaling in oxidative stressinduced liver injury. Semin. Liver Dis. 27:378-389.

48. Perl, A. 2005. Regulation of the pentose phosphate pathway and its impact on apoptosis under physiological and disease conditions. In Clinical and molecular aspects of defects in creatine and polyol metabolism. C. Jakobs and S. Stockler-Ipsiroglu, editors. SPS 
Publications. Heilbronn, Germany. 122-138.

49. Grossman, C.E., et al. 2004. Deletion of serine 171 causes misfolding, proteasome-mediated degradation, and complete deficiency of human transaldolase. Biochem. J. 382:725-731.

50. Price, N.E., and Cook, P.F. 1996. Kinetic and chemical mechanisms of the sheep liver 6-phosphogluconate dehydrogenase. Arch. Biochem. Biophys. 336:215-223.

51. Kaloyianni, M., and Kalomenopoulou, M. 1990. Regulation and rate of the hexose monophosphate shunt in Rana ridibunda erythrocytes. Comp. Biochem. Physiol. B. 95:287-294.

52. Dalton, T.P., Chen, Y., Schneider, S.N., Nebert, D.W., and Shertzer, H.G. 2004. Genetically altered mice to evaluate glutathione homeostasis in health and disease. Free Radic. Biol. Med. 37:1511-1526.

53. Will, Y., et al. 2000. gamma-glutamyltranspeptidase-deficient knockout mice as a model to study the relationship between glutathione status, mitochondrial function, and cellular function. Hepatology. 32:740-9.

54. Sochor, M., Kunjara, S., Greenbaum, A.L., and McLean, P. 1989. Changes in pathways of pentose phosphate formation in relation to phosphoribosyl pyrophosphate synthesis in the developing rat kidney. Effects of glucose concentration and electron acceptors. J. Dev. Physiol. 12:135-143.

55. Galvez, A.S., et al. 2003. Aldose reductase induced by hyperosmotic stress mediates cardiomyocyte apoptosis: differential effects of sorbitol and mannitol. J. Biol. Chem. 278:38484-38494.

56. Ramana, K.V., Friedrich, B., Bhatnagar, A., and Srivastava, S.K. 2003. Aldose reductase mediates cyto- toxic signals of hyperglycemia and TNF-alpha in human lens epithelial cells. FASEB J. 17:315-317.

57. Lornejad-Schafer, M.R., et al. 2005. Osmotic regulation of MG-132-induced MAP-kinase phosphatase MKP-1 expression in H4IIE rat hepatoma cells. Cell. Physiol. Biochem. 16:193-206.

58. Schliess, F., Heinrich, S., and Haussinger, D. 1998. Hyperosmotic induction of the mitogen-activated protein kinase phosphatase MKP-1 in H4IIE rat hepatoma cells. Arch. Biochem. Biophys. 351:35-40.

59. Kuntzen, C., et al. 2005. Inhibition of c-Jun-Nterminal-kinase sensitizes tumor cells to CD95induced apoptosis and induces G2/M Cell cycle arrest. Cancer Res. 65:6780-6788.

60. de la Coste, A., et al. 1998. Somatic mutations of the beta-catenin gene are frequent in mouse and human hepatocellular carcinomas. Proc. Natl. Acad. Sci. U. S. A. 95:8847-8851.

61. Miyoshi, Y., et al. 1998. Activation of the $\{$ beta $\}$ catenin gene in primary hepatocellular carcinomas by somatic alterations involving exon 3 . Cancer Res. 58:2524-2527.

62. Tannapfel, A., and Wittekind, C. 2002. Genes involved in hepatocellular carcinoma: deregulation in cell cycling and apoptosis. Virchows Arch. 440:345-352.

63. Leist, M., Single, B., Castoldi, A.F., Kuhnle, S., and Nicotera, P. 1997. Intracellular adenosine triphosphate (ATP) concentration: a switch in the decision between apoptosis and necrosis. J. Exp. Med. 185:1481-1486.

64. Picard, F., et al. 2002. Progesterone receptor knockout mice have an improved glucose homeostasis secondary to beta-cell proliferation. Proc. Natl. Acad.
Sci. U. S. A. 99:15644-15648.

65. Ishak, K.G., Goodman, Z.D., and Stocker, J.T. 1999. Tumors of the liver and intrahepatic bile ducts. Armed Forces Institute of Pathology. Washington, DC, USA. 356 pp.

66. Zhao, L., and Burt, A. 2007. The diffuse stellate cell system. J. Mol. Histol. 38:53-64.

67. Klatskin, G., and Conn, H.O. 1993. Histopathology of the liver. Oxford University Press. New York, New York, USA. 768 pp.

68. Carson, F.L. 1997. Histotechnology: a self-instructional text. American Society of Clinical Pathology. Chicago, Illinois, USA. 312 pp.

69. Berry, M.N., and Phillips, J.W. 2000. The isolated hepatocyte preparation: 30 years on. Biochem. Soc. Trans. 28:131-135.

70. Banki, K., Hutter, E., Gonchoroff, N., and Perl, A. 1999. Elevation of mitochondrial transmembrane potential and reactive oxygen intermediate levels are early events and occur independently from activation of caspases in Fas signaling. J. Immunol. 162:1466-1479.

71. Banki, K., Hutter, E., Colombo, E., Gonchoroff, N.J., and Perl, A. 1996. Glutathione levels and sensitivity to apoptosis are regulated by changes in transaldolase expression. J. Biol. Chem. 271:32994-33001.

72. Nagy, G., Koncz, A., and Perl, A. 2003. T cell activation-induced mitochondrial hyperpolarization is mediated by $\mathrm{Ca}^{2+}$ - and redox-dependent production of nitric oxide. J. Immunol. 171:5188-5197.

73. Grossman, C.E., Qian, Y., Banki, K., and Perl, A. 2004. ZNF143 mediates basal and tissue-specific expression of human transaldolase. J. Biol. Chem. 279:12190-12205. 